\title{
EFFETS DES EXTRACTIONS DE MATERIAUX ALLUVIONNAIRES SUR L'ENVIRONNEMENT AQUATIQUE DANS LES COURS SUPERIEURS DE LA LOIRE ET DE L'ALLIER
}

Synthèse des ètudes réalisèes de 1974 à 1976 par le Conseil Supérieur de la Pêche, 6e Délégation Régionale : Auvergne-Limousin - (1), avec l'aide financière :

- du Ministère de la Qualité de la Vie

- de l'Agence Financière de Bassin * Loire-Bretagne .

- du Fonds de gestion de la Taxe parafiscale sur les granulats

par

P. Clavel (2), R. CUINAT (3), Y. HAMON (4) et C. ROMANEIX (4) 1977

(1) 84, avenue du Puy-de-Dòme, 63000 CLERMONT-FERRAND.

(2) Docteur de $3^{\circ}$ cycle, université de Clermont-Ferrand.

(3) Délégué Régional du C.S.P.

(4) Ingénieur de IESITPA. 


\section{PLA N}

1 - RESUME 123

$\|$ - CIRCONSTANCES ET OBJET DE L'ETUDE ............. 123

III - Les gRavieres et les perturbations du BIOTOPE . . . . . 126

1 - Morphodynamique : modifications du lit, création ou aggravation d'obstacles ............................ 126

2 - Conséquences sur la physico-chimie de l'eau ........... 130

3 - Conséquences de la sédimentation des matières en suspension sur le fond du cours d'eau ...................... 130

IV - CONSEQUENCES SUR LES INVERTEBRES AQUUATIQUES ET SUR LES POISSONS

1 - Effets sur les macro-invertèbrès de fond ............. 134

a) Utilisation de la méthode des Indices Biotiques . . . . . . 134

b) Effets sur les densités numériques et sur les biomasses totales de macro-invertébrès ................. 135

c) Effets sur les densités et les biomasses de divers groupes d'invertèbrés ........................ 136

d) Effets sur la vitesse de colonisation du fond .......... 138

e) Conclusion sur les effets des gravières sur les invertébrés: notion de "pollution mécanique ".............. 138

2 - Effets sur les populations de poissons ............... 139

a) Reproduction : colmatage de frayères à truites ........ 139

b) Alimentation des poissons ................. 141

c) Inventaire pisciaire . . . . . . . . 142

$V \quad$ - LEGISLATION ET SOLUTIONS TECHNIQUES .............. 144

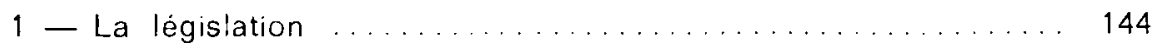

2 - La taxation par l'Agence Financière de Bassin ........... 144

3 - Les solutions techniques .................. 145

a) Suppression de la poilution par les eaux de lavage ..... 145

b) Problème des extractions dans le lit mineur du cours d'eau 146

VI - CONCLUSIONS $\ldots \ldots \ldots \ldots \ldots \ldots \ldots \ldots \ldots \ldots \ldots \ldots$

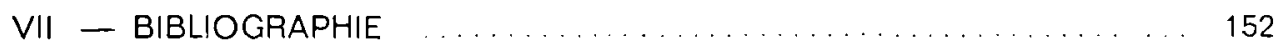




\section{I - RESUME}

De 1974 à 1976, l'Allier et la Loire ont été étudiés dans les départements de la Haute-Loire et du Puy-de-Dôme, au niveau d'une vingtaine d'exp!oitations de granulats, opérant ou non dans le lit mineur et $y$ rejetant ou non des eaux après lavage. de $8 \mathrm{~km}$.

Les modifications du lit ont été mesurées par écho-sondage sur un parcours

Ont également été étudiées, par échantillonnages répartis sur une année et de l'amont à l'aval de plusieurs exploitations :

- la physico-chimie de l'eau (notamment matières en suspension);

- les modifications de la granulometrie du fond (notamment par substrats artificiels) ;

- les modifications de la faune benthique, par méthodes "qualitatives " (indices biotiques) et quantitatives (deisités et biomasses des divers groupes d'invertébrés) ;

- les populations de poissons (inventaires par pêche électrique) et leur régime alimentaire, à l'amont et à l'aval d'une exploitation sur la Loire;

- la survie d'œufs de salmonides déposés à l'amont et à l'aval d'une exploitation sur l'Allier.

Toutes ces observations confirment que la plupart de ces extractions provoquent une "pollution mécanique" dont les effets s'accentuent par accumulation de l'amont à l'aval, dans l'Allier. Des moyens techniques simples pour limiter ces nuisances sont proposés.

\section{II - CIRCONSTANCES ET OBIET DE L'ETUDE}

L'extraction de matériaux alluvionnaires - sable, gravier, cailloux est-elle pratiquement sans effets sérieux sur l'environnement aquatique et la pêche, comme l'ont généralement soutenu, jusqu'à ces dernières années, la plupart des responsables de cette industrie? Ou constitue-t-elle une nuisance réelle, comme le soutiennent de plus en plus les collectivités de pêcheurs. notamment dans les vallées de la Loire supérieure, de l'Allier et du Cher, où ces activités sont intenses?

Pour tenter de répondre objectivement à cette question, une étude a été confièe, de 1974 à 1976, à la Région Piscicole "Auvergne-Limousin ", par le Conseil Superieur de la Pêche, le Ministère de la Qualité de la Vie, la Taxe Parafiscale sur les Granulats et l'Agence de Bassin "Loire-Bretagne".

Le présent article vise à en résumer les résultats qui ont déjà été présentés en détail dans divers rapports précédents $(8,9,10,27)\left({ }^{*}\right)$.

\section{*****}

La "production" de granulats en Auvergne (Cantal. Haute-Loire, Puy-deDôme et Allier) est passée de 3,7 millions de tonnes en 1969 à plus de 6 millions en 1974 (20). Le département du Puy-de-Dôme entre pour $65 \%$ dans cette production: les besoins en matériaux de viabilité, notamment, y sont très importants.

( $\left.{ }^{*}\right)$ Les nombres entre parentheses renvoient à la bibliographie (chapitre VII). 


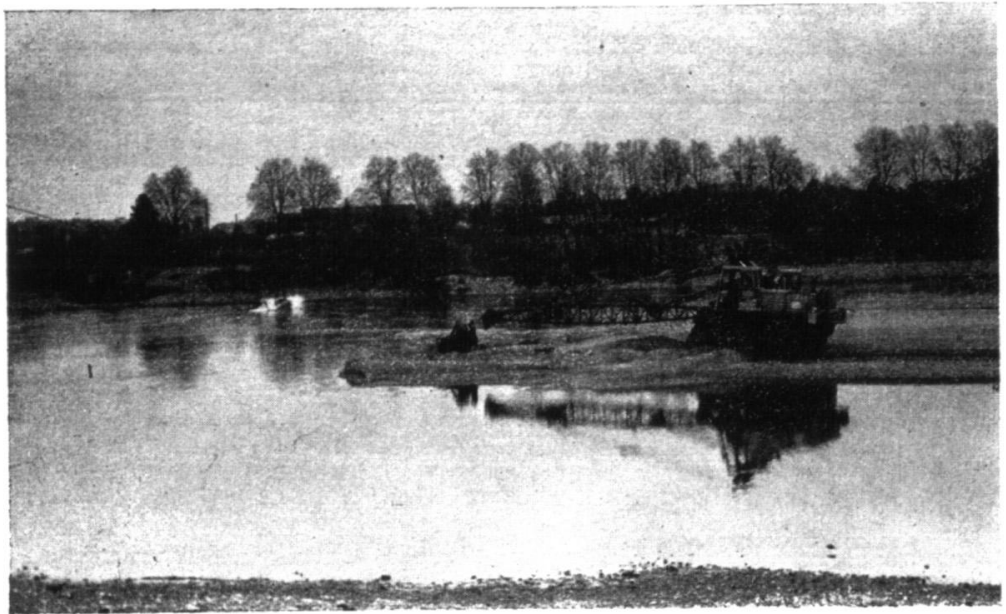

Photo 1 : EXPLOITATION DANS LE LIT MINEUR DE L'ALLIER, à l'aval de Moulins (mars 1976), par drag-line sur toute la largeur de la rivière, et par godet opérant sur un banc d'aggrégats.

La production en Haute-Loire est stagnante, voire en diminution; les réserves alluvionnaires se présentant sous forme de "poches " peu étendues, alors qu'en Puy-de-Dôme, en bordure de l'Allier, les terrasses alluviales sont généralement larges et épaisses, ce qui en rend l'exploitation plus aisée (7).

Nous avons recensé, en 1976 :

- dans le département de la Haute-Loire : 24 exploitations, opérant presque toutes dans le lit mineur de l'Allier ou de la Loire;

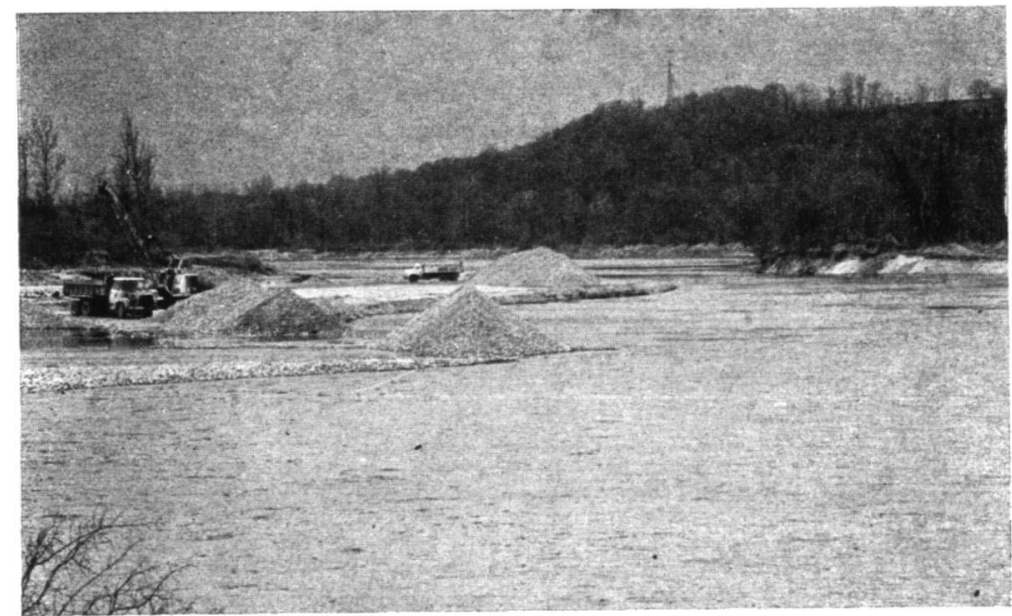

Photo 2 : EXPLOITATION ET DEPOT PROVISOIRE D'AGGREGATS DANS LE LIT MINEUR DE L'ALLIER (Puy-de-Dôme, 1975).

- dans le département du Puy-de-Dôme : 51 exploitations, dont 15 extrayant dans le lit mineur de l'Allier ou de la Dore.

Les extractions dans le département de l'Allier, également très nombreuses, n’ont pas été recensées. 
Selon les conditions d'extraction, dans l'eau ou à sec, les procédés et gins utilisés sont différents. Les matériaux sont gènéralement lavés et triés $r$ des tamis. L'eau utilisée (en moyenne $0,5 \mathrm{~m}^{3}$ par $\mathrm{m}^{3}$ de matériaux extraits) $t$ généralement pompée dans la riviere ou dans la nappe. Elle est ensuite letée, soit dans une ancienne gravière, soit le plus souvent dans la rivière, rfois après transit dans des bassins de décantation généralement sousnensionnés et rarement curès.

\section{**}

Les cours d'eau - Loire et Allier - ont été étudiés à l'amont et à l'aval une vingtaine d'exploitations; certaines zones ont fait l'objet d'observations irticulièrement poussées, quant au nombre de points de prélèvements ou expérimentation, ou à l'échelonnement des échantillonnages dans le temps, afin $\geq$ couvrir une année complète (fig. 1).

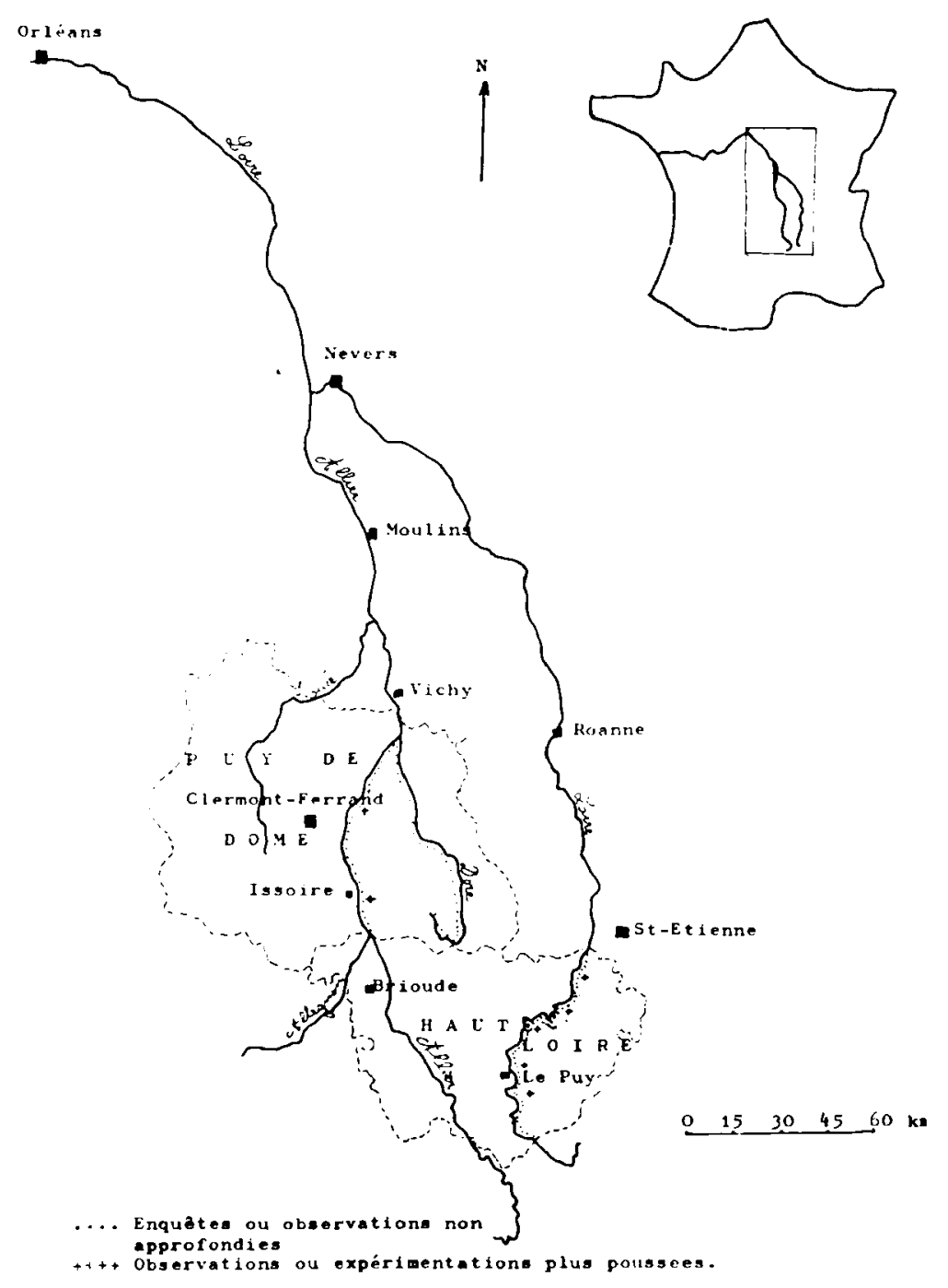

Fig. 1 : SITUATION GEOGRAPHIQUE DE L'ETUDE 
Les techniques d'étude utilisées sont décrites dans le rapport détaillé (10). Elles ne sont que très brièvement citées dans la présente synthèse, au niveau des divers chapitres qui concernent successivement :

- les perturbations du biotope, résultant du dragage ou du rejet des eaux de lavage ;

- les répercussions sur les organismes aquatiques (invertébrés, poissons) ;

- les aspects juridiques et les moyens techniques de réduire ces perturbations.

\section{III - LES GRAVIERES ET LES PERTURBATIONS DU BIOTOPE}

Dans le Puy-de-Dôme, trois cas ont ètè ètudiès, sur l'Allier : les gravières MAIA, en 1975, et particulièrement Julhiard, en 1975-76, près d'lssoire, ainsi qu'un secteur en cours de rectification à l'aval de Clermont-Ferrand.

En Haute-Loire, six gravières ont éiè ètudiées en 1974, la gravière Gillet, en amont du Puy-en-Velay, ayant constitué le terrain d'expérimentation par la suite (1975-76).

Nous avons essayé de mesurer les perturbations du biotope en considérant trois aspects :

- Rectifications de l'Allier sur 4 km entre Pont-du-Château et Joze (63) : Différences morphodynamiques entre une zone témoin (non draguée) et une zone soumise aux extractions.

- Modifications physico-chimiques de l'eau, à l'aval de plusieurs gravières (sur la Loire et l'Allier).

- Colmatage du fond de la rivière par les particules minérales (limons) rejetées ou remises en suspension par le dragage.

1 - Morpho-dynamique : modifications du lit, création ou aggravations d'obstacles.

74 profils en travers, réalisés à l'aide d'un écho-sondeur (") sur l'ensemble du secteur, aboutissant aux résultats résumés ci-après (voir aussi exemple à la figure 2).

(*) Appareil prèté par le CTGREF de Bordeaux. 


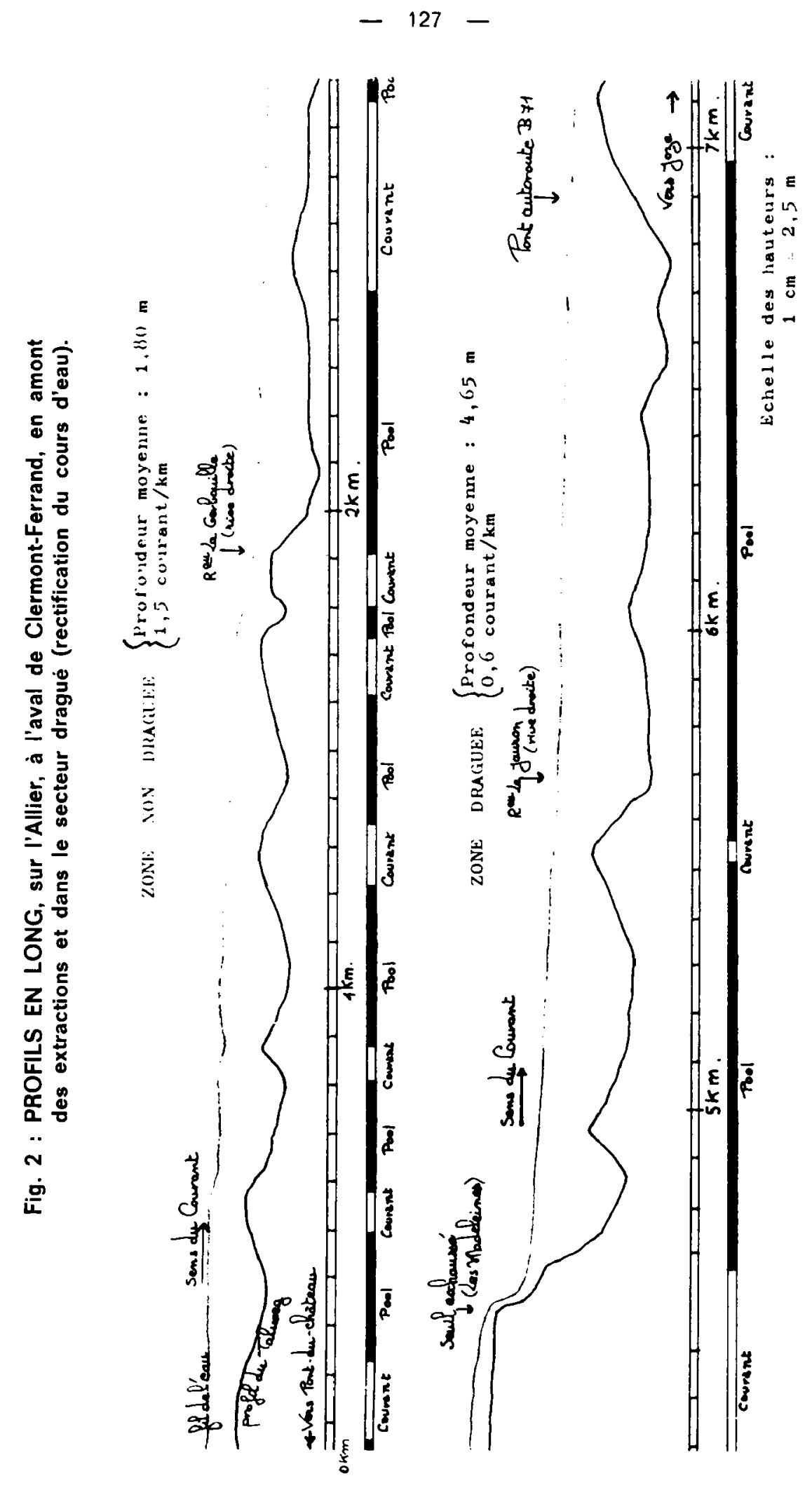




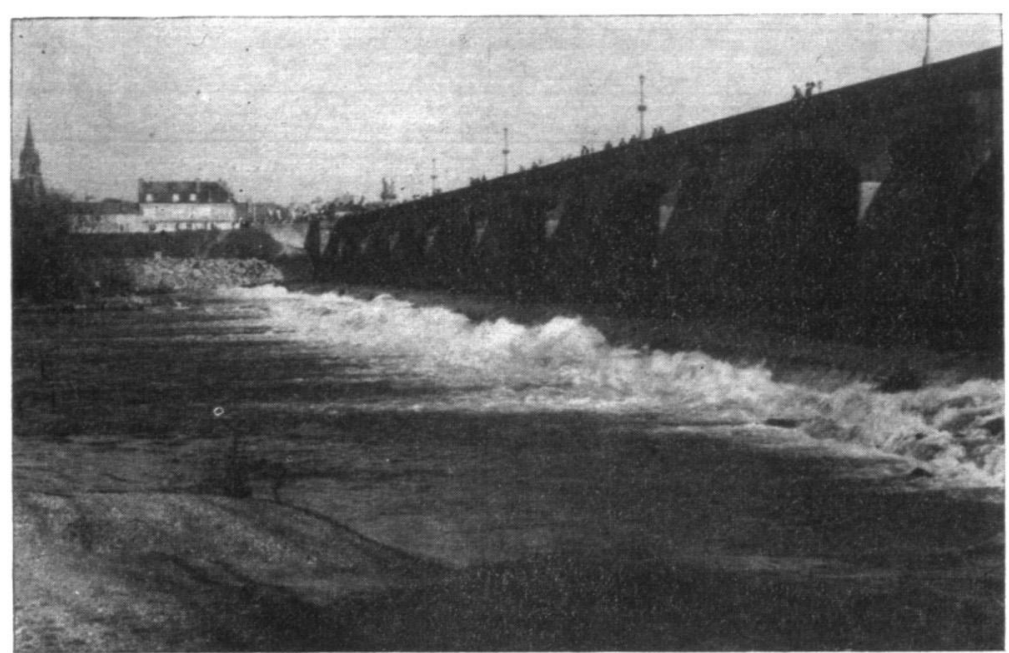

Photo 3 : LE PONT REGEMORTES A MOULINS (03), SUR L'ALLIER, en mars 1976.

Le seuil, affouillè par le surcreusement du lit, constitue, en basses eaux, une chute d'environ $1,5 \mathrm{~m}$, difficilement franchissable par les poissons, notamment le Saumon.

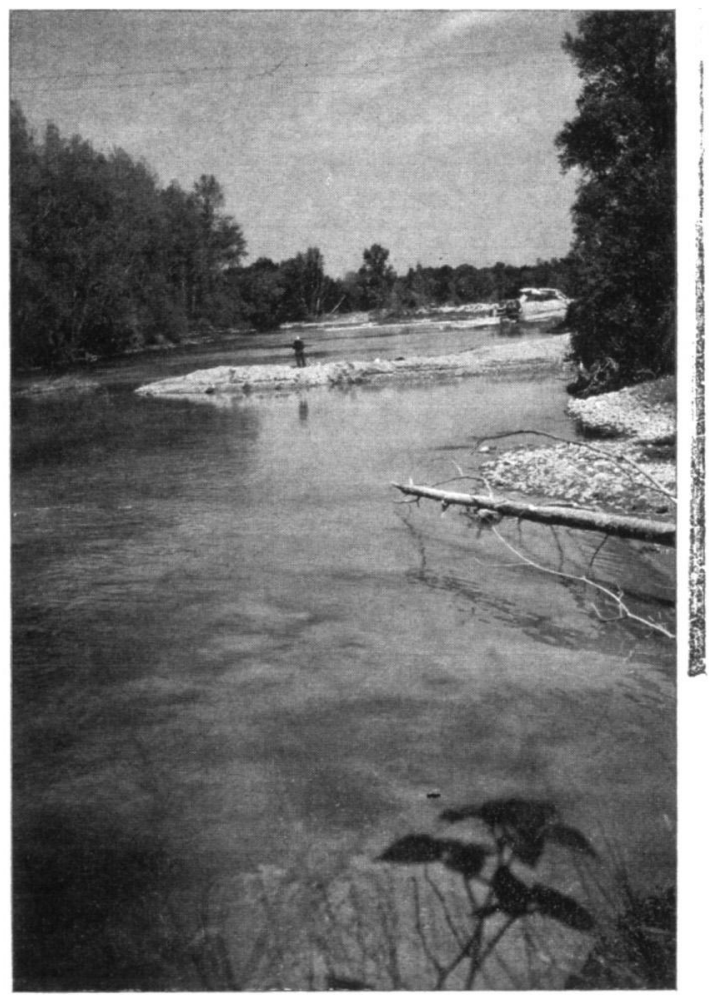

Photo 4 : ASPECT DE L'ALLIER DANS UNE ZONE D'EXTRACTION D'AGGREGATS DANS LE LIT MINEUR (Puy-de-Dôme, juillet 1976). L'eau est brune sur toute la largeur du cours d'eau. 


\section{- Zone témoin (non draguée) : 4,6 km}

Profondeur moyenne : $1,80 \mathrm{~m}$;

alternance de 8 pools et 7 courants $(1,5$ courant $/ \mathrm{km})$;

dissymetrie du profil en travers ;

berges boisées, herbiers nombreux; relative stabilité du milieu.

Cette zone se terminait à l'aval par un seuil marneux. Peu apparent d'abord, il est apparu de plus en plus " exhaussé * relativement au reste du lit, affouillé par les extractions réalisées à l'aval. En 1975, la chute atteignant 1,5 m, jointe aux blocs de marne encombrant le lit, s'est avérée infranchissable par la majoritè des poissons, notamment par le saumon. Des explosifs et de gros engins ont été nécessaires pour améliorer cette situation.

\section{- Zone draguée : $3,2 \mathrm{~km}$}

A l'aval de la chute. les extractions par draglines ont entrainé une uniformisation du milieu :

profondeur moyenne : $4,65 \mathrm{~m}$;

seulement 3 grands pools et 2 courants $(0,6$ courant $/ \mathrm{km})$;

fond et berges instables, végétation arrachée, accès au cours d'eau rendu difficile ou dangereux.

Nous observons une baisse du niveau typologique théorique (méthode définie en 1974 : 35) entre le secteur témoin et le secteur remaniè (10).

L'apparition ou l'exhaussement de seuils n'a pas été observée qu'à Pont-du-Château, mais en plusieurs points où sont maintenant perturbées les remontées des migrateurs (Saumon, cyprinidés, carnassiers), surtout par débit moyen à faible, ou par température basse, notamment :

- seuil du pont de Guétin (58);

- seuil du pont de Règemortes, à Moulins (03) ;

- seuil du barrage mobile de Vichy (03);

- seuil du barrage mobile de Pont-du-Château (63) ;

- barrages de la Bajasse (à Brioude) et de Langeac (43).

Les extractions tendent également, maintenant, à perturber les migrations des poissons de la façon indirecte suivante : en surcreusant le lit. les exploitations ont pour effet d'abaisser le niveau moyen des nappes aquifères voisines.

- Dans la région de St-Germain-des-Fossés - Billy (03), le tarissement des puits filtrants, en 1976, a conduit le Syndicat d'adduction d'eau à établir d'urgence un barrage en palplanches et enrochements de 1,5 m de hauteur.

- Dans la zone de rectification et d'extractions liée à la construction de l'autoroute B 71, l'édification de "seuils " est apparue nécessaire, notamment pour conserver le niveau des nappes.

Mème si chacun d'eux était équipé du dispositif de franchissement approprié (échelle à poissons), il est certain que la multiplication de ces nouveaux ouvrages, s'ajoutant à l'aggravation des anciens par * exhaussement relatif", ralentit sérieusement la migration du Saumon, parfois même l'interdit totalement et risque d'aboutir à courte échéance à l'extinction de l'espèce (14). 
Bien que non abordées dans cette étude, les conséquences de ces surcreusements sur la tenue de divers ouvrages d'art (ponts, barrages) nous semblent également préoccupantes.

\section{2 - Conséquences sur la physico-chimie de l'eau.}

Dès 1974, les mesures effectuees sur la Loire indiquaient que la plupart des paramètres physico-chimiques de l'eau n'étaient pas affectés à l'aval des gravières: des constatations identiques ont èté faites expérimentalement au Canada (28).

Seules, Turbidité et Matières en suspension (MES = Matières minérales + faible pourcentage de matière organique) augmentaient à l'avai des exploitations. Nous avons estimè à 0.7 tonne (poids sec) la quantite de MES rejetóes dans la Loire, en 8 heures de fonctionnement de la gravière Gillet (*), pour un débit prélevé d'eau de lavage de $100 \mathrm{~m}^{3} / \mathrm{h}$.

La gravière Julhiard, sur l'Allier, déversait entre 0.3 et 3,8 tonnes de limons par jour ; 99 pour cent de ces MES sont constitués de particules de taille inférieure à 200 microns

Le dragage lui-même dans le lit mineur de la Loire (gravière Gillet) met en circulation 6,5 tomnes de "fines" par jour $1 \mathrm{~km}$ à l'aval de l'extraction (pour un débit de la rivière de $6 \mathrm{~m}^{3} / \mathrm{s}$ ).

Un à deux kilomètres à l'aval d'une gravière, le taux de MES est encore sensiblement affecté, mais la sedimentation des particules les plus grossières est rapide et, au-delà de deux kilomètres, seule la turbidité (due à la fraction la plus fine) reste élevée, en période de débit moyen à faible $\left(^{* *}\right)$. La succession d'un grand nombre de ces rejets aboutit, par accumulation, à une déterioration caractérisée de la clarté et de la valeur piscicole de l'Allier dans ses cours moyen et surtout inférieur (31)

\section{3 - Conséquences de la sédimentation des matières en suspension sur le fond du cours d'eau.}

Pour mesurer le dépôt des limons à l'aval des extractions, nous avons utilisé des plaques en ciment de $0.1 \mathrm{~m}^{2}$ sur lesquelles ètaient fixés des cailloux (une poche de plastique enroulée sur les bords pendant l'immersion permettait de récupérer la totalité du dépôt lors du relevé). Ces plaques ont été immergées en faciès lentique (courant moyen : $0.4 \mathrm{~m} / \mathrm{s}$ ), au niveau de la gravière Gillet. sur la Loire, et relevées au bout de 1 mois, 3 mois et 5 mois.

Trois stations ont èté étudiées : amont de la gravière, $500 \mathrm{~m}$ aval, et $800 \mathrm{~m}$ aval (fig. 3).

(*) Cette gravière traitait environ 650 tonnes de granulats par jour.

(*) L'augmentation de la teneur en M.E.S. dans le cours d'eau atteint, à l'aval de certaines gravières:

- 100 et parfois $130 \mathrm{mg} / \mathrm{l}$ a $50 \mathrm{~m}$;

- quelques unités à $30 \mathrm{mgll}$ à $1000 \mathrm{~m}$

Le F.A.O. considère que les eaux contenant plus de $80 \mathrm{mg} / \mathrm{l}$ de M.E.S. ne peuvent fournir une bonne production piscicole (15). 


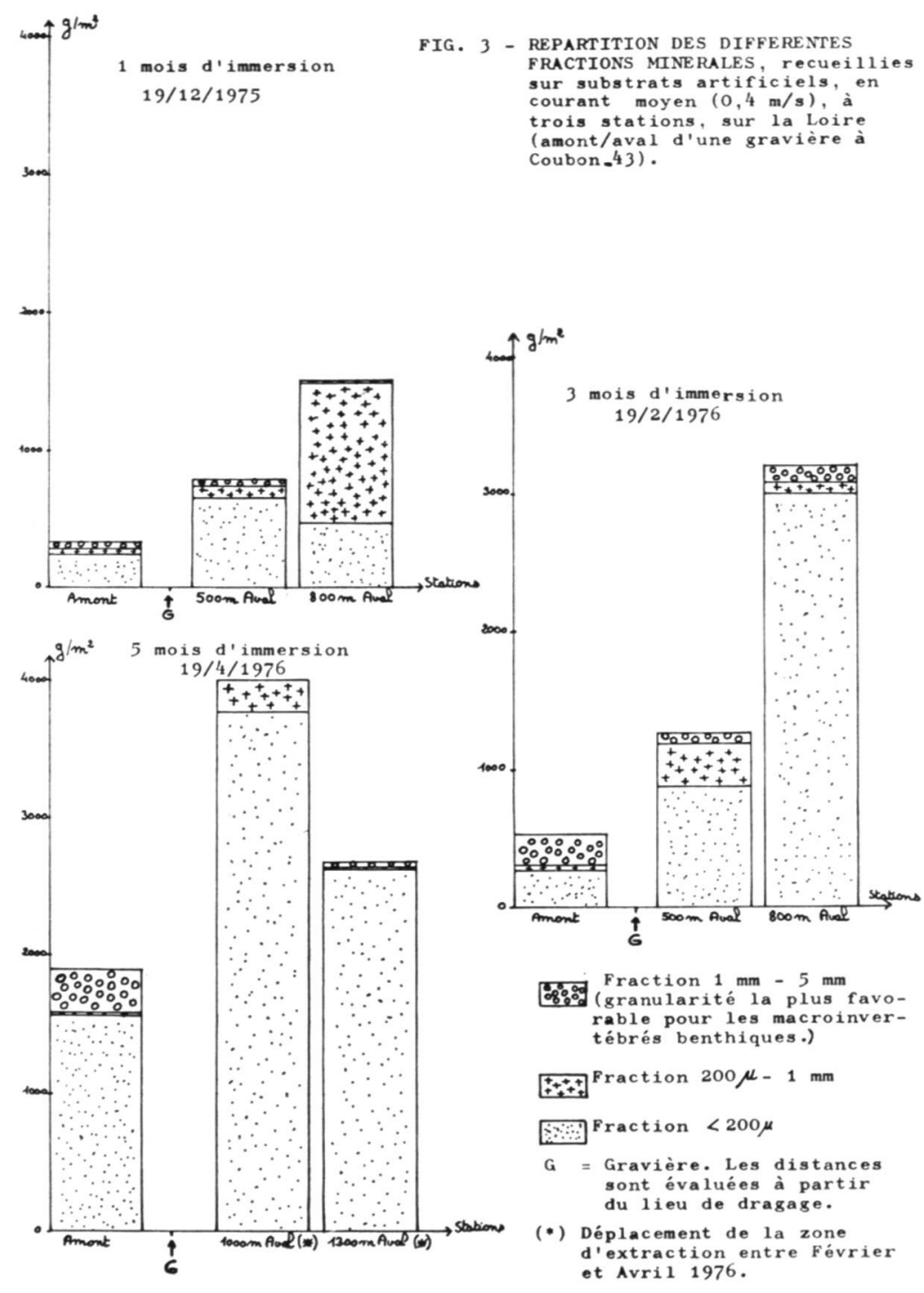

Fig. 3 : REPARTITION DES DIFFERENTES FRACTIONS MINERALES, recueillies sur substrats artificiels, en courant moyen $(0,4 \mathrm{~m} / \mathrm{s})$, à trois stations, sur la Loire (amont/aval d'une gravière à Coubon - 43).

Les dépôts recueillis sont une résultante des lavages et décantations successifs dus aux modifications continuelles des courants, du débit, et aux déplacements de la zone d'extraction.

Parmi les 3 fractions retenues:

- $5 \mathrm{~mm}-1 \mathrm{~mm}$ (gravette) (1), 


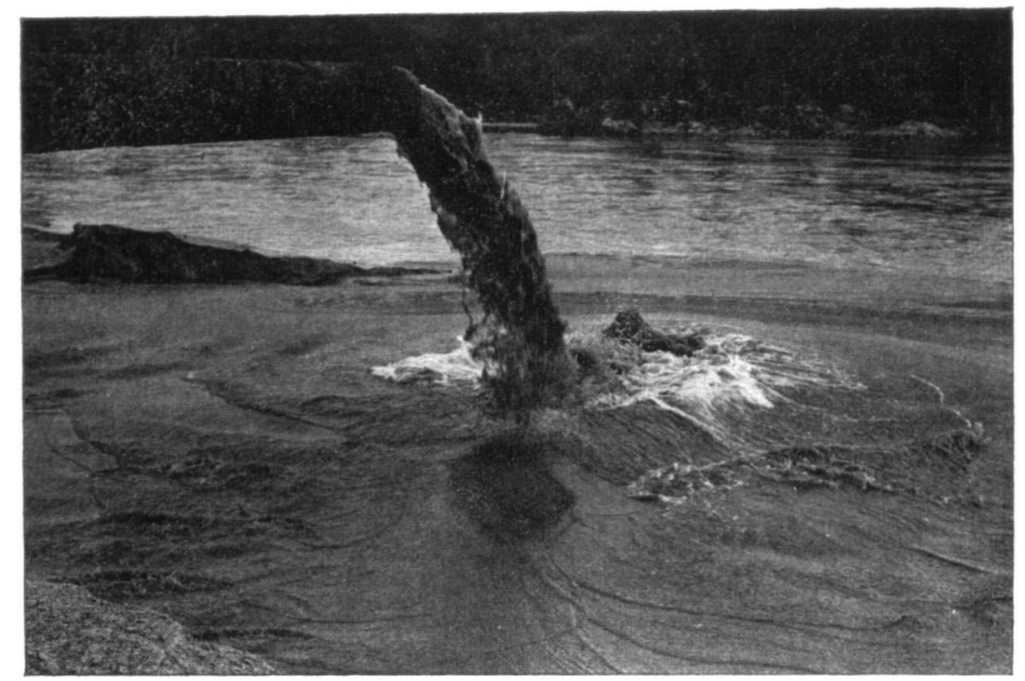

Photo 5 : UN REJET DIRECT D'EAU DE LAVAGE DES AGGREGATS DANS LA LOIRE (Cher, nov. 1976).

Malgré la très importante dilution, le cours d'eau est troublé sur toute sa largeur, après mélange, quelques centaines de mètres à l'aval.

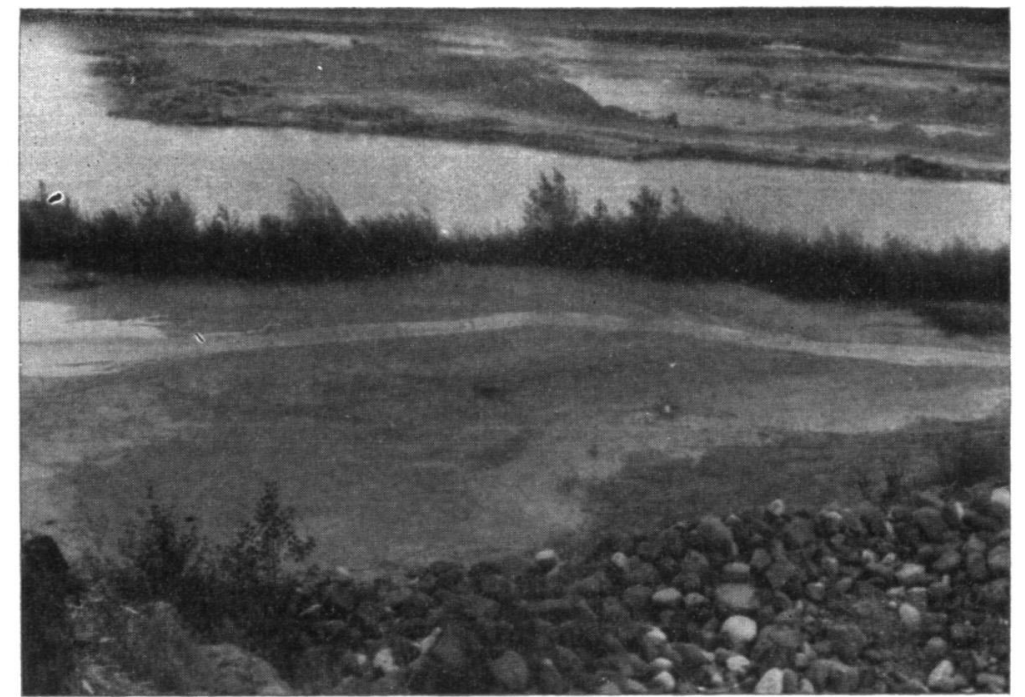

Photo 6 : UN BASSIN DE DECANTATION D'EAU DE LAVAGE, ENTIEREMENT COLMATE après quelques mois de fonctionnement, sur la Loire (Haute-Loire, 1975).

Le lit de la Loire, bouleversé par d'anciennes extractions, est visible en contre-bas (arrière-plan). 
- $1 \mathrm{~mm}$ - 200 microns (sable) (2).

- inférieur à 200 microns (sablon, limon, argile) (3),

les classes (2) et (3) sont plus abondantes (en poids) aux stations aval : l'amont.

fraction inférieure à 200 microns : multiplièe par 1,7 à 11 par rapport à

fraction comprise entre $1 \mathrm{~mm}$ et 200 microns : multipliée par 1,2 à 25 .

La fraction * gravette*, la plus favorable aux macro-invertébrés $(21,32)$, est peu représentée à l'aval : divisée par 2,7 à 10 . Elle disparait même, $500 \mathrm{~m}$ à l'aval, en avril.

A l'aval de cette exploitation, comme de plusieurs autres observées sur la Loire ou l'Allier, le colmatage du fond est très sensible. surtout dans les zones à courant faible à moyen, sur des distances atteignant 0,5 à 2 kilomètres.

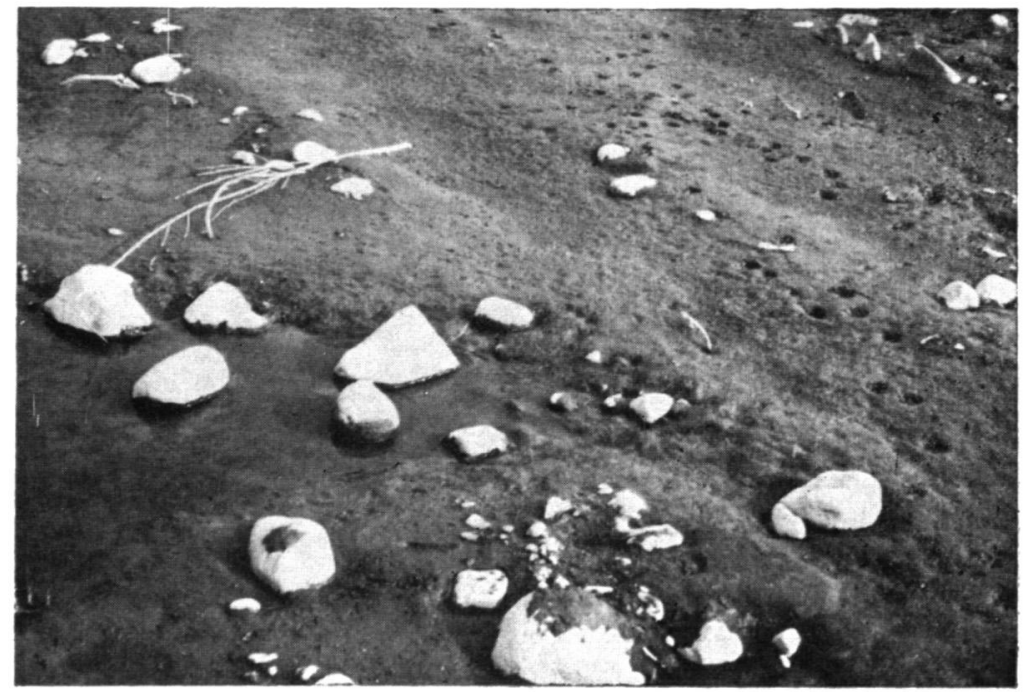

Photo 7 : ASPECT DU FOND DU COURS D'EAU, EN PERIODE DE FAIBLE DEBIT, A L'AVAL DE DEUX GRAVIERES (Loire, Bas-en-Basset, HauteLoire, été 1974).

Seuls les gros cailloux émergent du dépôt de limon et sont eux-mêmes recouverts d'une pellicule qui blanchit en séchant.

La plupart des invertébrés aquatiques ont disparu de cette zone, sauf les vers oligochètes, très abondants.

\section{IV - CONSEQUENCES SUR LES INVERTEBRES AQUATIQUES ET SUR LES POISSONS}

Les modifications du milieu (morphodynamique, qualité de l'eau, nature du fond) que nous avons observées ont-elles des effets sur les organismes vivants dans le cours d'eau? Nos études ont surtout porté sur les populations de macro-invertébrès benthiques, constituant à la fois des * indicateurs de qualité du milieu * et la base de la nourriture des poissons. Des observations quantitatives sur les populations de poissons et sur leur reproduction n'ont pu être menées que dans une zone plus restreinte. 


\section{1 - Effets sur les macro-invertébrès de fond.}

Les prélèvements de benthos ont été effectués à l'amont et à l'aval des gravières, à l'aide d'un filet échantillonneur de fond de $1 / 10 \mathrm{~m}^{2}$ (Filet de Surber) à vide de maille de 250 microns, à raison de deux prélèvements par faciès (iotique : courant rapide; lentique: courant faible).

Pour certaines gravières, les échantillonnages ont porte sur des substrat, artificieis (plaques en ciment, voir III. 3) ou des "substra's naturels reconstitués" (cagettes de $0,2 \mathrm{~m}^{2}$ remp'ies de cailloux et graviers), préalabiement mis en place pour faciliter les études.

Le benthos de taille superieure à $5 \mathrm{~mm}$ a èté triè à part. L'èchantillon (avec invertébrés inférieurs à $5 \mathrm{~mm}$ ) était débarrassé des éléments minéraux (sable) puis fractionné.

Les invertébrés ont été triés, dèierminés (généralement jusqu'au genre $\left({ }^{*}\right.$ ). comptès et pesés (après séchage à $105^{\circ} \mathrm{C}$ ).

\section{a) Utilisation de la méthode des Indices Biotiques.}

Mise au point en Grande-Bretagne, en 1964, par WOODIWISS (38), elle était essentiellement utilisée pour détecter des pollutions organiques: elle a été adaptée par TUFFERY et VERNEAUX, qui ont établi un tableau type (33)

Schématiquement, cette méthode conduit à attribuer une note (IB) de 10 à 0 , selon qu'un milieu est non pollué à très gravement pollué. Cette note dépend à la fois

- de la présence ou non de "groupes faunistiques" d'invertébrés, classés selon leurs "exigences" vis-à-vis de la qualité du milieu (ou de leur * intolérance " à la plupart des pollutions, notamment organiques);

- du nombre total "d'unités systèmatiques" (généralement genre, ou famille) trouvées.

Il est à noter que, dans ce système, c'est la présence ou l'absence des Unités Systématiques qui conditionne la note, mais que le nombre (ou densité numérique) d'individus de chaque Unité Systématique déterminée n'influe pas.

Cette méthode a été utilisée en 1974 dans l'étude du profil de qualité de la Loire en Haute-Loire, sur un parcours de $96 \mathrm{~km}$ :

- Elle a mis en évidence, sur $30 \mathrm{~km}$ de cours à l'ava! de l'agglomération du Puy, un secteur de poliution organique importante (IB $<5$, au lieu de $\mathrm{IB}=8$ à 9 dans la zone amont), suivi d'une zone régénèrée $(\mathrm{IB}=8$ à 9$)$, fortement eutrophisée sur $40 \mathrm{~km}\left({ }^{*}\right)$.

- Elle n'a pas mis en évidence de chute sensible ( $>1$ à 2 points) de l'amont à l'aval de chacune des gravières étudiées, que celles-ci se situent dans la zone organiquement polluée ou non.

(*) Le Laboratoire de Zoologie de la Faculté de Clermont Ferrand, ainsi que Monsieur A. MARTY, de la Région Piscicole, nous ont aidé pour certaines déterminations.

$\left({ }^{*}\right)$ Dans cette dernière zone, la diversité faunistique (nombre d'unités systématiques différentes) était maximale, avec apparition d'éphéméroptères * fouisseurs *. 
D'auires études, en 1975 et 76 sur la Loire et sur l'Allier, niont pas non p'us montré d'infiuence sur les indices biotiques, même à l'aval immédiat $(50 \mathrm{~m})$ de la gravière.

Ainsi, les indices biotiques s'avèrent très peu sensibles aux effets des gravières, du moins avec les dilutions observées dans des cours d'eau de l'importance de la Loire et de l'Allier.

Il en est de même de "l'indice de diversité" de SHANNON et WEAVER (30), qui tient compte de l'abondance et de la diversité du benthos et caractérise le degré de stabilité des communautés de macro-invertébrés.

Le fait que ces deux indices ne soient pas, ou que très peu, affectés par les gravières provient essentiellement :

- de ce que l'ordre de sensibilité des divers invertébrès aux matières en suspensions n'est pas le même que dans les autres pollutions, organiques notamment ;

- et surtout de ce que le nombre des unités systématiques n'est pratiquement pas affecté à l'aval d'une gravière.

Par contre, la densité numérique des invertébrés s'avère profondément modifiée, comme indiqué au paragraphe suivant.

b) Effets sur les densités numériques et sur les biomasses totales de macro-invertébrés. gravières:

Les mesures faites sur la Loire et sur l'Allier ont révélé, à l'aval des

- une réduction très sensible de la densité numérique totale d'invertébrés (diminution de 13 à $75 \%$ ); (réduction également constatée par d'autres auteurs : 16,28 ) ;

- une réduction aussi sensible de la biomasse totale de ces invertébrés (diminution de 10 à $81 \%$ ).

Précisons notamment que, dans la zone d'extraction située en amont du Puy, particulièrement étudiée à diverses époques de l'année, c'est en avril que la biomasse d'invertébrés était maximale; elle atteignait alors environ $600 \mathrm{~kg}$ par hectare, en amont, contre environ $120 \mathrm{~kg} / \mathrm{ha}$, un kilomètre en aval de la gravière (Gillet).

Ces résultats globaux ne traduisent que très grossièrement l'effet des gravières ètudiées : si la plupart des catégories d'invertébrés sont raréfièes, quelques autres voient par contre leur densité accrue.

c) Effets sur les densités et les biomasses de divers groupes d'invertébrés.

La comparaison entre les invertébrés aquatiques à l'amont et à l'aval d'une des exploitations ètudiées est représentée graphiquement aux figures 4 et 5 . 


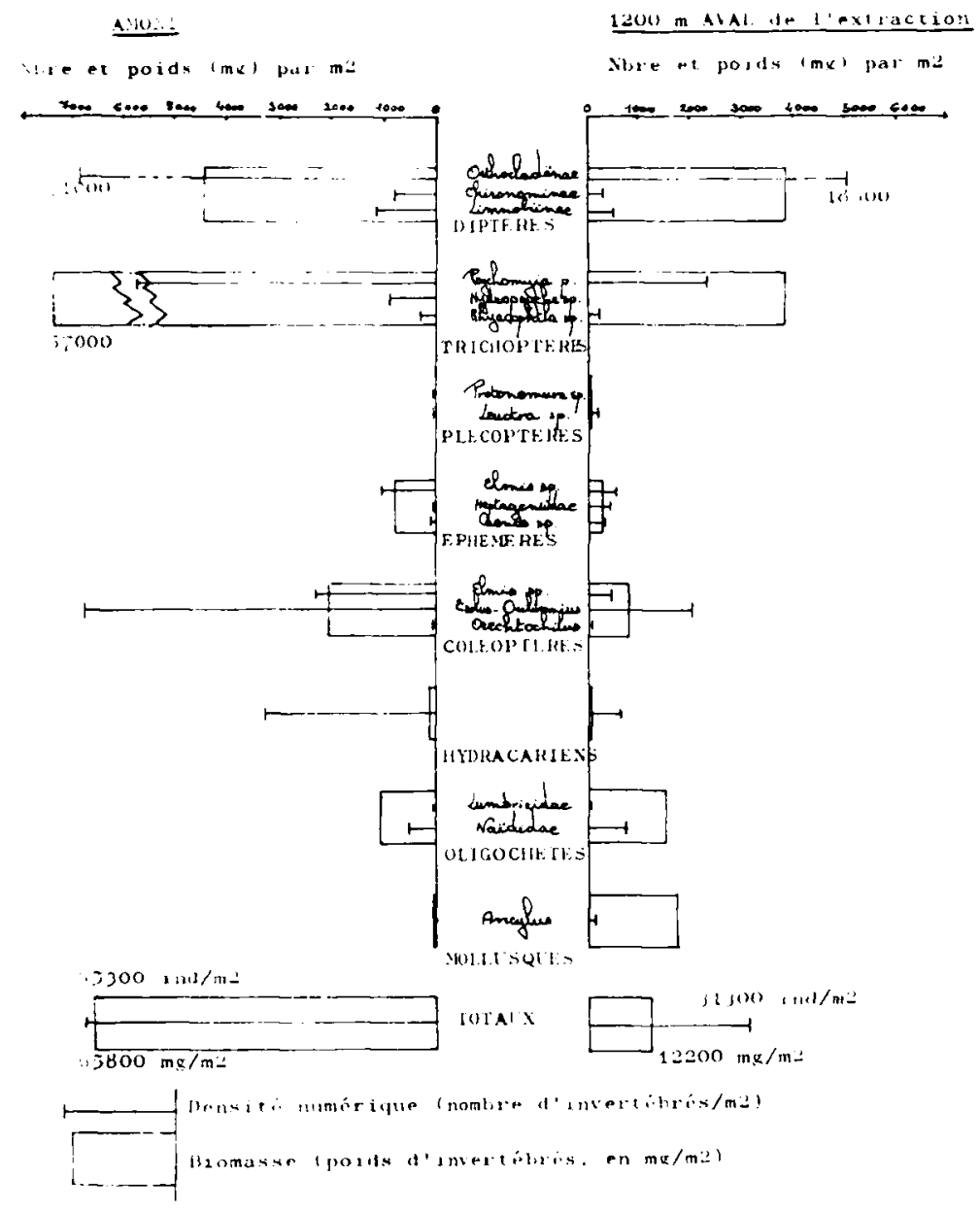

Fig. 4 : DENSITES ET BIOMASSES D'INVERTEBRES BENTHIQUES, à l'amont et à l'aval (1 $200 \mathrm{~m}$ ) d'une gravière, sur la Loire, à Coubon (43), en avril 1976. FACIES LOTIQUE (Courant)

Le faciès lotique représente environ $80 \%$ de la surface des stations étudiées. 


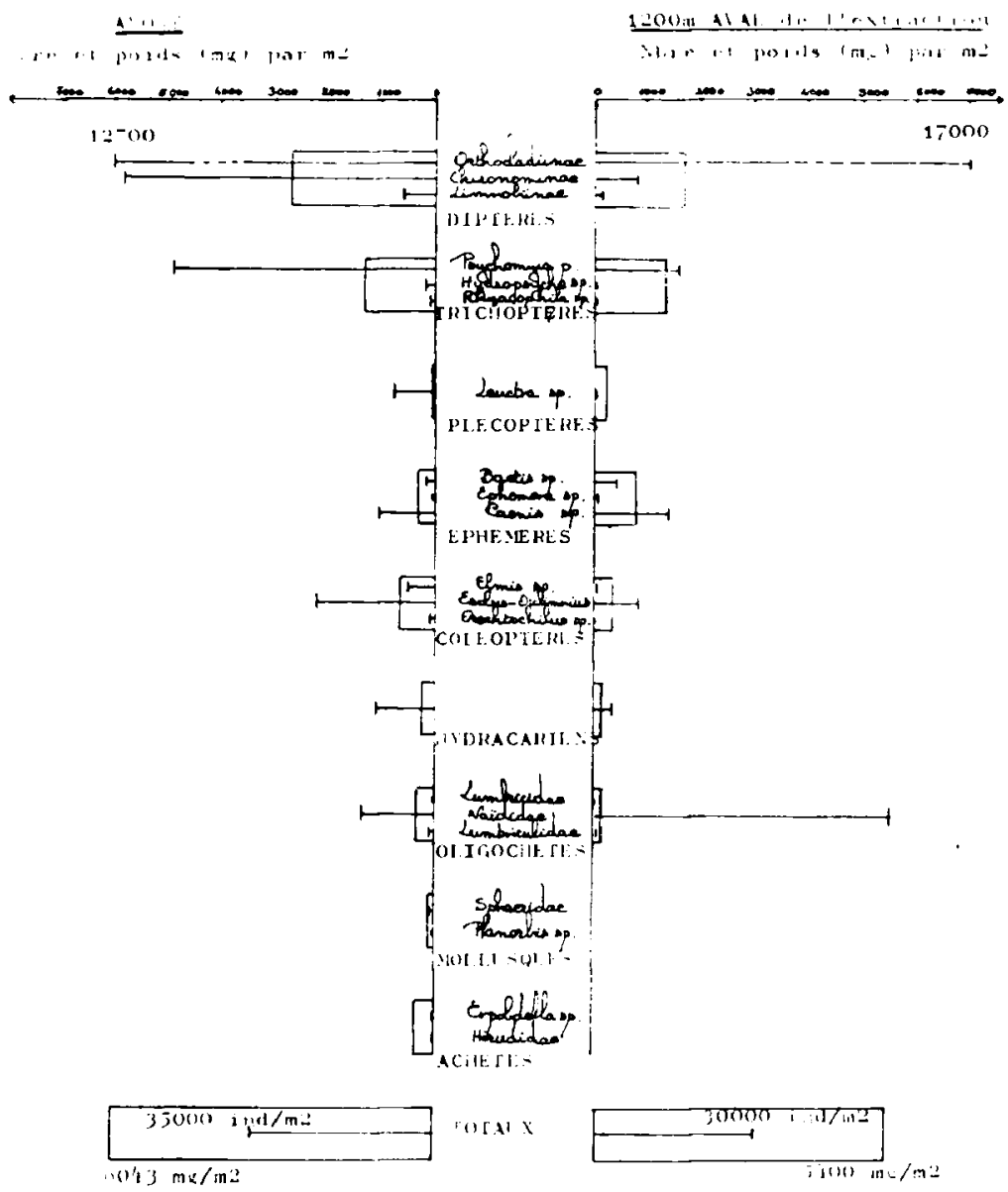

Fig. 5 : DENSITES ET BIOMASSES D'INVERTEBRES BENTHIQUES, à l'amont et à l'aval (1 $200 \mathrm{~m})$ d'une gravière, sur la Loire, à Coubon (43) en avril 1976. FACIES LENTIQUE (CaIme)

D'une façon générale, sur la Loire supérieure et l'Allier, certains groupes d'invertébrés voient presque toujours leur abondance réduite à l'aval des gravières :

- Dipteres : Chironominae, Orthocladiinae, Limnobiinae, Simuliidae;

- Trichopteres : Psychomya pusilla, Rhyacophila, Mystacides, Oligoplectrum maculatum, Hydropsyche (");

- Coléoptères : Elmis, Esolus-Oulimnius ;

- Epheméroptères : Torleya belgica:

- Crustacés : gammarus (à noter que ce genre disparait presque toujours à laval des gravières (*);

(*) Egalement constaté sur des cours d'eau anglais (19). 
- Hydracariens ;

- Plécoptères : Leuctra, en faciès lentique. au total.

Cette réduction affecte ainsi 15 unités systématiques sur 87 recensées

II est à noter que les deux groupes Dipteres et Trichoptères

- constituent en général près de 80 pour cent du nombre tota! d'invertébrés du fond de ces rivières,

- y jouent un rôle majeur dans l'alimentation des poissons (voir IV 2 b).

Quelques groupes voient au contraire leur abondance accrue (en valeur absolue) à l'aval des gravières

- Oligochètes : Lumbriculidae et Tubificidae; ces invertébrés sont considérés comme fouisseurs, dans la vase et notamment la matière organique.

- Megaloptère : Sialis, également fouisseur : n'a èté observé qu'à l'aval des gravières.

- Epheméroptère : Caenis ( ${ }^{*}$ ).

L'étude des contenus stomacaux a montré que ces groupes d'invertébrés étaient très peu consommés par la plupart des espèces de poissons (barbeaux, vandoises, chevaines, ablettes).

Les autres groupes d'invertébrés voient leur abondance tantôt réduite, tantôt augmentée à l'aval des diverses gravières étudiées. Parmi eux, seuls les Baetis (Ephéméroptères) tiennent une place importante dans le benthos et les estomacs de poissons, dans les deux cours d'eau.

\section{d) Effets sur la vitesse de colonisation du fond.}

Les études sur substrats artificiels ont montré, de plus, que la colonisation par les invertébrés s'effectuait plus rapidement à l'amont (où elle semblait terminée au bout de 14 jours) qu'à l'aval (terminée au bout d'un mois).

Ces résultats semblent directement liés au fait que ces substrats aval étaient recouverts assez rapidement par du limon ( $<200 \mu$, voir III 3 ).

e) Conclusion sur les effets des gravières sur les invertébrés : notion de "pollution mécanique ".

Les résultats résumés ci-dessus permettent de conclure que les communautés d'invertébrés aquatiques, à l'aval des gravières, sont profondément modifiées

- augmentation de l'abondance de quelques groupes, adaptés à des milieux vaseux ou limoneux (vers oligochètes, sialis et caenis), mais généralement peu consommés par les poissons ;

- diminution des groupes d'invertébrès habituellement les plus représentés dans le milieu (sauf Baetis) et dans l'alimentation des poissons;

(*) A noter que la présence d'éphéméroptères suffit, dans la méthode des indices biotiques, à décerner une note assez élevée (et même très élevée dans le cas de l'éphéméroptère Ecdyonurus). Or, Caenis et Ecdyonurus se sont avérés non affectés par les matières en suspension. 
- diminution souvent importante du nombre et du poids total d'invertébrés par unité de surface du fond (réduction de 10 à $80 \%$ de la biomasse totale).

Ces modifications s'observent sur des distances pouvant atteindre deux kilomètres, à l'aval de certaines gravières, ce qui est considérable pour des cours d'eau de l'importance de la Loire et de l'Allier.

Elles aboutissent à réduire la diversité et surtout l'abondance de la nourriture disponible pour les poissons (voir IV 2 b). Ceci, joint aux effets possibles sur la reproduction de ces poissons (voir IV 2 a), montre bien que les augmentations des matières en suspension, liées à la plupart de ces gravières, constituent bien une "pollution", qualifiée par de précédents auteurs (notamment VIBERT et LAGLER, 1961 ; NISBET, non publié) de a pollution mécanique •.

Contrairement aux pollutions organiques, les pollutions mécaniques n'augmentent pas la biomasse d'invertébrés $\left({ }^{*}\right)$, comme l'ont prouvé les études sur la Loire qui est affectée par les deux types de pollution.

Agissant essentieliement sur l'habitat et sur les possibilités de développement de la plupart des invertébrés, sans en interdire totalement la présence, ce type de pollution ne s'avère pas décelable ni chiffrable (sauf dans des cas extrêmes) par la méthode des indices biotiques, méthode adaptée essentiellement aux pollutions organiques (et éventuellement chimiques).

Des méthodes basées sur l'évaluation des densités (ou biomasses) de diptères et trichoptères (ou de certaines catégories d'entre eux) sembleraient mieux adaptées à caractériser rapidement ces pollutions mécaniques.

II est probable aussi que le nouveau système d'indices biotiques, actuellement mis au point au CTGREF par VERNEAUX et ses collaborateurs; sera plus sensible à ce type de pollution.

En attendant, l'évaluation de la note de "capacité biogénique ", d'après la méthode de LEGER (22), reste la méthode nous paraissant la mieux adaptée.

\section{2 - Effets sur les populations de poissons.}

Nous avons essayé de mettre en évidence les effets des pollutions mécaniques sur le niveau trophique supérieur constitué par les poissons.

Les aspects "Reproduction" et "Alimentation" ont été considérés, ainsi que la densité et la structure des populations pisciaires à l'amont et à l'aval d'une graviere.

\section{a) Reproduction : colmatage de frayères de truites.}

Répartis en plusieurs lots, 15000 œufs embryonnés de truites (Salmo trutta fario) ont été enfouis, en boites Vibert, sous graviers, en courant rapide $(0,8 \mathrm{~ms} /)$ et en courant moyen $(0,2 \mathrm{~m} / \mathrm{s})$, à l'amont, à $150 \mathrm{~m}^{(* *)}$ et à $750 \mathrm{~m}\left(^{* *}\right)$ à l'aval du rejet de la gravière Julhiard, sur l'Allier.

(*) Dans le cas des pollutions organiques, les biomasses importantes sont dues aux oligochètes et aux diptères chironomidae, la diversité faunistique étant par ailleurs très réduite, ce qui n'est pas le cas a laval des gravieres.

$\left({ }^{*}\right) 150 \mathrm{~m}$ en aval, la station est fortement soumise aux M.E.S. (turbidité de l'eau multpliée par 2, par rapport à lamont). $750 \mathrm{~m}$ en aval, la dispersion des M.E.S. est totale, leur concentration n'ost supérieure que de quelques milligrammes par rapport à la station témoun.

Pendant loxposition dos cufs. le rejet de la gravière était relativement peu chargé en M.E.S. (2800 mgli) par comparaison avec dautres périodes où sa concentration dépassait $16000 \mathrm{mgll}$ : Son débit étart denvirun $50 \mathrm{~m}^{3} / \mathrm{h}$; celui de lAllier a varié de 60 à $120 \mathrm{~m}^{3} / \mathrm{s}$. 
Une mortalité moyenne des œufs et alevins, variant de 75 pour cent, $150 \mathrm{~m}$ à l'aval, à 65 pour cent, à $750 \mathrm{~m}$, a été observée. Le taux de mortalité dans la station témoin (amont) était d'environ 20 pour cent (fig. 6).
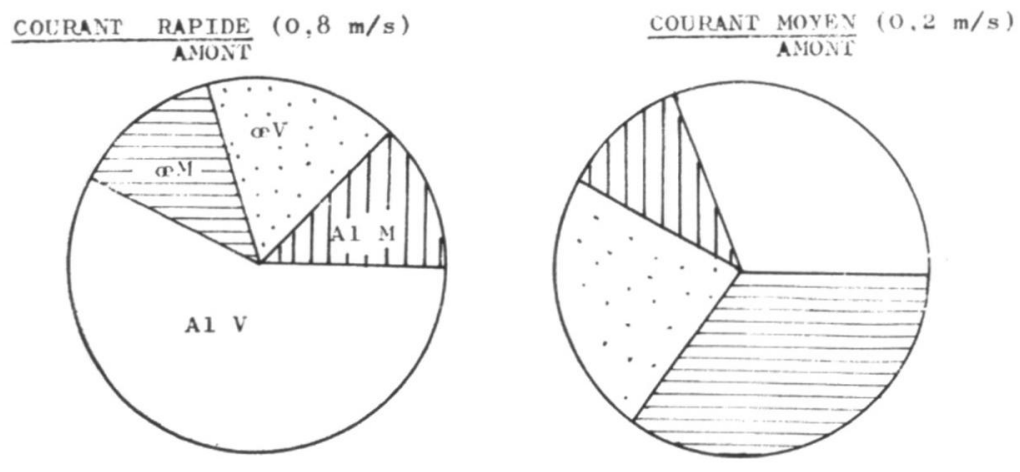

1 o m AVAL du rejet

150 m AVAL du rejet
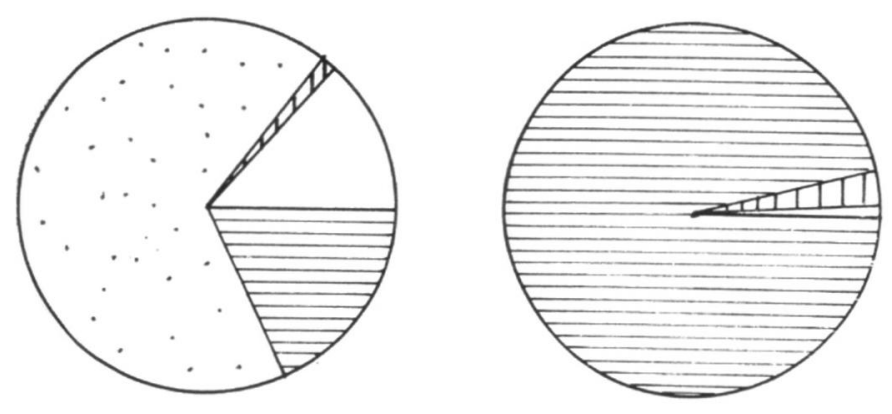

750 m AVAL du rejet

750 m AVAL dil rejet
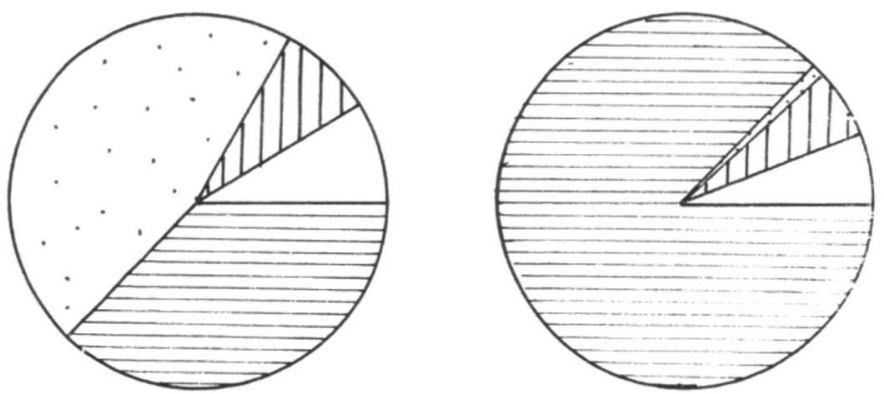

Fig. 6 : INFLUENCE DES M.E.S., sur des œufs (œ) et alevins (AI) de truites, sur l'ALLIER, au niveau d'une gravière, près d'ISSOIRE, le 1 er mars 1976, après 20 jours d'immersion.

$V=$ Vivant, $M=$ Mort (voir légende sur cercle en haut à gauche). 
Par ailleurs, la durée d'incubation parait augmenter dans les deux stations aval, ce qui entraine une exposition plus longue des œufs aux MES, et peut expliquer partiellement l'augmentation des mortalités. 13, 37)

Ce colmatage des frayères a èté également citè par divers auteurs (12,

L'effet des matières en suspension sur les œufs de cyprinides (déposés sur des supports divers) n'a pas èté étudié ici. II l'a été par le CTGREF (DQEPP).

\section{b) Alimentation des poissons.}

Dans deux secteurs de la Loire, à l'amont et à l'aval $(800 \mathrm{~m})$ de la gravière Gillet (amont du Puy), les contenus stomacaux de 137 poissons (essentiellement barbeaux et vandoises) ont été étudiés, en septembre 1975 et avril 1976 (figure 7).

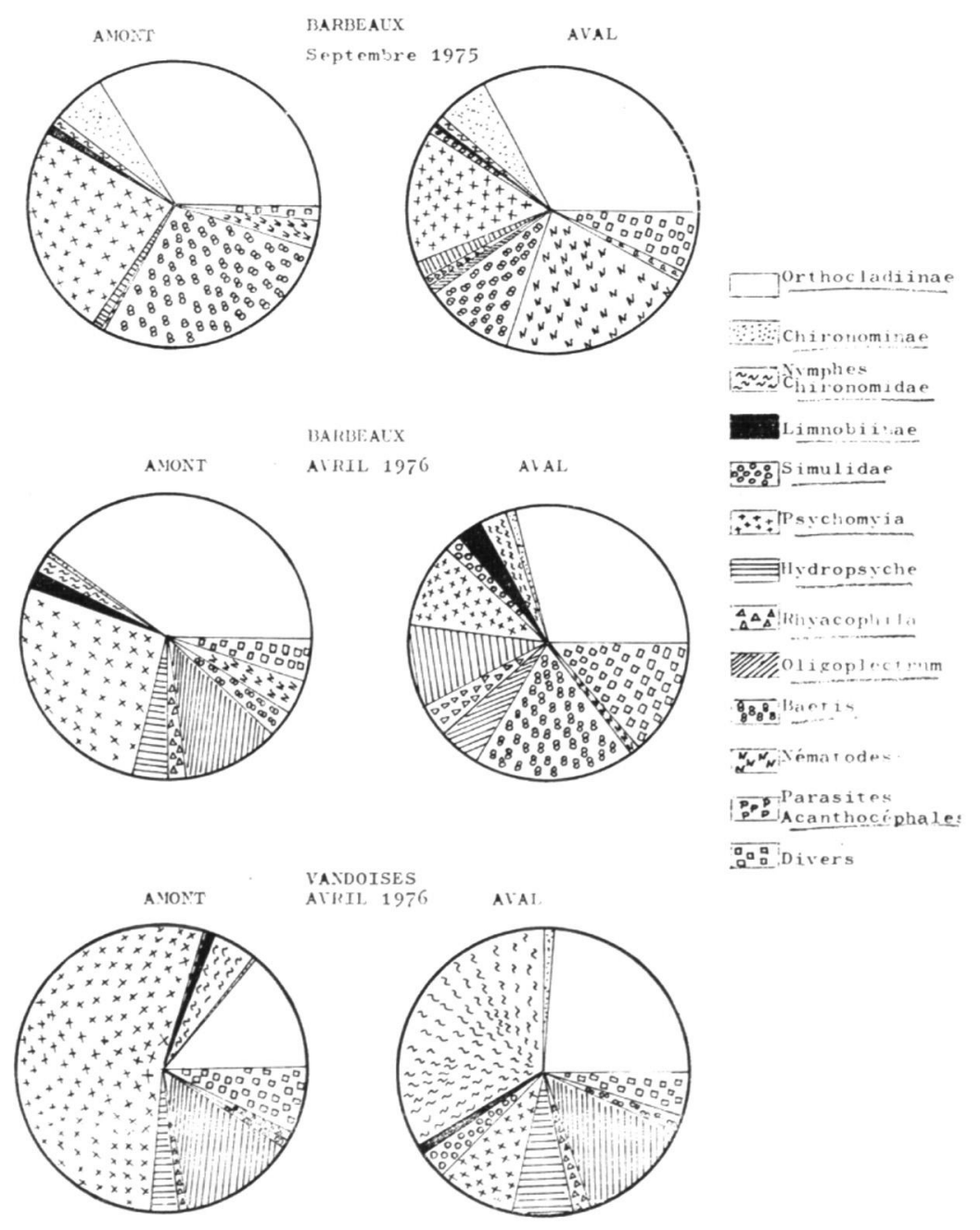

Fig. 7 : COMPOSITION MOYENNE DES CONTENUS STOMACAUX DE POISSONS CAPTURES A L'AMONT ET $800 \mathrm{M}$ A L'AVAL D'UNE GRAVIERE, SUR LA LOIRE, A COUBON (43). 
Une quarantaine de macro-invertébrés différents a été trouvée au total.

Les groupes Diptères. Trichoptères et Ephémères constituent $99 \%$ de l'alimentation de ces poissons, aussi bien à l'amont qu'à l'aval de l'exploitation. Nous avons vu (IV 1 c) que ces deux premiers groupes apparaissaient réduits dans le benthos à l'aval des gravières.

Chez les Barbeaux de septembre, prédominent le diptère Orthocladiinae. le trichoptère Psychomyia pusilla et l'éphèméroptère Baetis.

En moyenne, Psychomyia et Baetis sont présents en moindre quantité dans les estomacs des poissons pêchés en aval, ou les Nématodes et les parasites Acantocephales sont par contre plus abondants (ces derniers n'ayant pas été observés dans les contenus stomacaux amont).

Chez les Barbeaux d'avril, prédominent Orthocladiinae, Psychomyia pusilla, Baetis, Oligoplectrum maculatum et Hydropsyche.

En moyenne, Orthocladiinae, Psychomyia pusilla, Oligoplectrum maculatum sont moins représentés dans les poissons ava!; mais Hydropsyche et Baetis y sont plus nombreux.

Chez les Vandoises pêchées en avril, les groupes dominants sont Psychomyia pusilla, Orthocladiinae, Chironomidae (Nymphes) et Oligoplectrum maculatum.

En moyenne, Psychomvia pusilla est moins abondant dans les estomacs aval; Orthocladiinae. Nymphes de chironomidae y étant mieux représentés qu'à l'amont.

Cette première étude de contenus stomacaux :

1) ne met pas en évidence, de façn statistiquement significative de différences importantes dans le régime alimentaire, c'est-à-dire le choix des proies entre les poissons de l'amont et ceux de l'ava! de la gravière ;

2) nous permet, par contre, de connaitre les catégories d'invertébrés les plus recherchées par les poissons, aussi bien à l'amont qu'à l'aval : Psychomyia, Orthocladiinae, Baetis, Oligoplectrum et Hydropsyche, principalement.

Or, nous avons vu (IV 1 c) que toutes ces catégories, à l'exception de Baetis, voient le plus souvent leurs effectifs réduits à l'aval des gravières. Quant aux quelques Unités Systématiques apparemment favorisées par les dépôts de limon (Oligochètes, Caenis. Sialis). elles n'apparaissent qu'exceptionnellement (Sialis chez un seul poisson) dans les contenus stomacaux.

De ces deux constatations. (1) et (2), on peut actuellement conclure que les rejets de matières en suspension, en réduisant la quantité de nourriture utilisable par les poissons (barbeaux et vandoises, étudiés ici), peuvent constituer indirectement un facteur limitant dans la croissance et l'abondance de ces poissons.

\section{c) Inventaire pisciaire.}

Les résultats des pêches électriques effectuées en amont et $800 \mathrm{~m}$ en aval de la gravière Gillet sur la Loire, montrent une diminution de 28 pour cent de la Densité numérique totale des poissons, ainsi qu'une réduction de leur Biomasse de l'ordre de $17 \%$, à l'aval, par rapport à l'amont de l'extraction.

L'ensemble des espèces est affecté (principalement les ablettes qui diminuent de 99 pour cent, les vandoises de 93 pour cent, les vairons de 68 pour 
cent et les goujons de 48 pour cent), à l'exception des chevesnes (augmentation de 31 pour cent) et des barbaaux (augmentation de 124 pour cent) (voir figure 8).

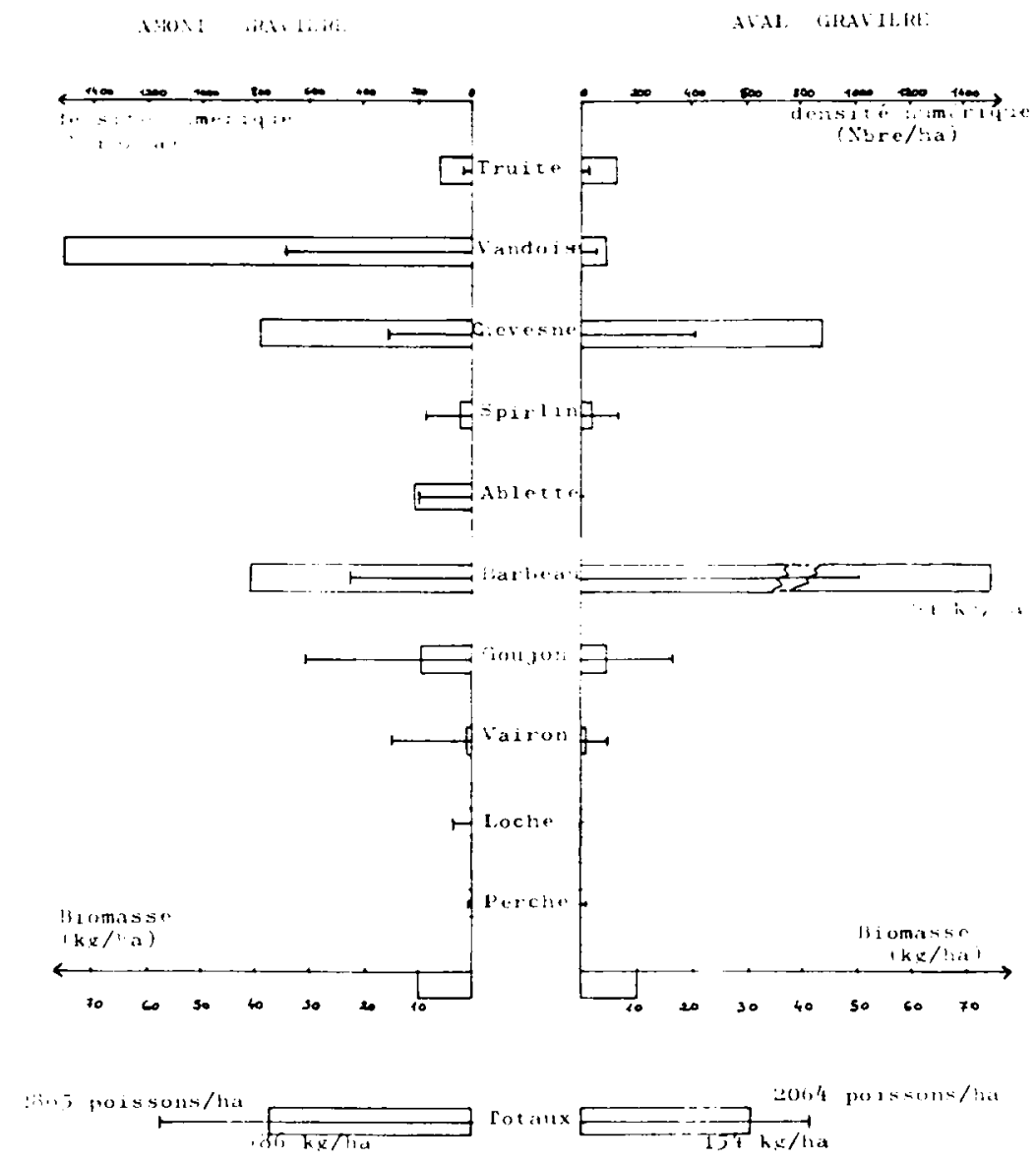

Fig. 8 : POPULATION ICHTYAIRE ESTIMEE EN AMONT ET 800 M EN AVAL DE LA GRAVIERE GILLET (04-09-1975).

La structure de la population est donc modifiè à l'aval, les barbeaux représentant 52 pour cent dı peuplement total dans ce secteur, contre 16 pour cent à l'amont et 3 espèces constituant 83 pour cent de l'effectif aval contre 5 espèces à l'amont.

Des espèces de faible intérêt halieutique sont favorisées (barbeaux) au détriment des truites et ablettes notamment.

La réduction de l'importance et la modification de la structure spécifique du peuplement en poissons, à l'aval de la gravière, peut s'expliquer :

- d'une part du fait d'une action directe des matières en suspension sur les œufs et les alevins de certaines especes,

- d'autre part du fait de la réduction de la nourriture disponible, comme vu au paragraphe précédent. 
Il n'a pas èté possible, dans la présente étude, d'évaluer la distance jusqu'à laquelle se font encore sentir les effets de cette gravière sur la population de poissons. Des précisions sur ce point seraient plus faciles à obtenir sur des cours d'eau de moindre importance que la Loire ou l'Allier.

\section{V - LEGISLATION ET SOLUTIONS TECHNIQUES}

Ces atteintes au milieu aquatique ne sont pas inéluctables; trois types de moyens sont disponibles pour les limiter : législation, taxation et solutions techniques.

\section{1 - La législation.}

Sur ce chapitre, que nous ne pouvons que résumer très brièvement ici, on se reportera utilement au rapport lui-même (10). Les textes législatifs en vigueur, et notamment les décrets du 20 septembre 1971, renforcent le pouvoir et les responsabilités des administrations compétentes (DDE, DDA. Service des Mines).

D'autre part, l'arrêté interministériel du 13 mai 1975 fixe les conditions techniques auxquelles sont subordonnés les rejets. L'article 7 précise, par exemple, que le rejet ne doit pas provoquer une coloration visible du milieu récepteur, ce qui interdit donc les déversements augmentant sa turbidité.

L'article 434-1 du Code Rural est également applicable aux pollutions mécaniques, puisque les matières en suspension provenant de l'activité d'une gravière nuisent à la nutrition et à la reproduction du Poisson. Enfin, le Code du Domaine Public fluvial (circ. 15-9-72) interdit aux exploitations hors du lit d'extraire à moins de $11,70 \mathrm{~m}$ des berges.

La législation parait donc suffisante. En fait, elle a été jusqu'ici peu ou pas appliquée, comme nous l'avons constaté dans cette étude. II en est souvent de même du cahier des charges de beaucoup d'exploitations, faute probablement pour les Administrations de disposer de moyens de contrôle suffisants sur le terrain.

Doit-on aussi expliquer cette situation par le fait que les Directions Departementales de l'Equipement, qui sont automatiquement consultées par le Service des Mines pour réglementer ces exploitations, sont en même temps les plus gros clients, directs ou indirects, de ces exploitations qui leur fournissent des matériaux de viabilité ? D'autres facteurs, sortant du cadre de cette étude, sont aussi probablement la cause de cette situation que déplorent actuellement les collectivités piscicoles.

\section{2 - La taxation par l'Agence Financière de Bassin.}

Elle concerne les prélèvements d'eau et les rejets. Cette imposition semble dissuasive sur ce point, mais toutes les exploitations ne sont pas encore recensées par l'Agence et les contrôles du bon fonctionnement des bassins de décantation sont rares. Ces bassins sont généralement déclarés par l'exploitant à l'Agence, ce qui réduit ou supprime la taxation sur le rejet; mais leur entretien laisse à désirer et, très souvent, les eaux de lavage ne font que traverser les bassins comblés, atteignant donc la rivière sans épuration.

Par contre, il n'existe pas de taxation de l'opération de dragage ellemême, dans le lit mineur. Or, cette études a montré que l'incidence, sur l'envi- 
ronnement aquatique, de ce type d'extraction était aussi importante, sinon plus, que les rejets d'eau de lavage.

\section{3 - Les solutions techniques.}

a) Suppression de la pollution par les eaux de lavage.

Il est techniquement très facile de retenir, par décantation, la majorité des déchets de lavage : dimensionné pour un temps de séjour moyen de six heures, un bassin de decantation retient 96 pour cent environ des matières en suspension, pour les effluents de nature peu argileuse.

Toutefois, notre étude a montré que cette seule solution était rarement suffisante, dans la pratique, pour la protection du cours d'eau :

- la plupart des bassins de décantation observés sont comblés après quelques semaines ou mois de fonctionnement et deviennent absolument inopérants, faute d'entretien;

- même à la sortie de bassins bien dimensionnés et entretenus, les eaux de lavage contiennent encore les matieres en suspension les plus fines: elles colmateront peu le fond du cours d'eau, mais augmenteront encore sérieusement sa turbidité.

Beaucoup plus sûr est donc le lavage en circuit fermé, comme nous l'avons vu pratiquer avec succès par quelques exploitants. Les eaux chargèes par le lavage, après transit dans deux bassins de décantation, conçus pour être alternativement mis à sec et curés facilement, sont réutilisées

- ce système, qui réduit considérablement les taxations sur l'eau (voir $\S$ précédent), donne des garanties plus sérieuses sur l'entretien des bassins;

- il supprime le rejet au cours d'eau des particules les plus fines; celui-ci n'est donc pas troublé; il semble, par ailleurs, que ces particules ne gênent pas le lavage et n'alterent pas la qualité des granulats obtenus.

Les boues retirées des bassins de décantation sont parfois utilisables en agriculture. Mais le plus souvent elle peuvent servir à combler danciens trous d'extraction, ce qui limitera les atteintes au paysage en fin d'exploitation. 


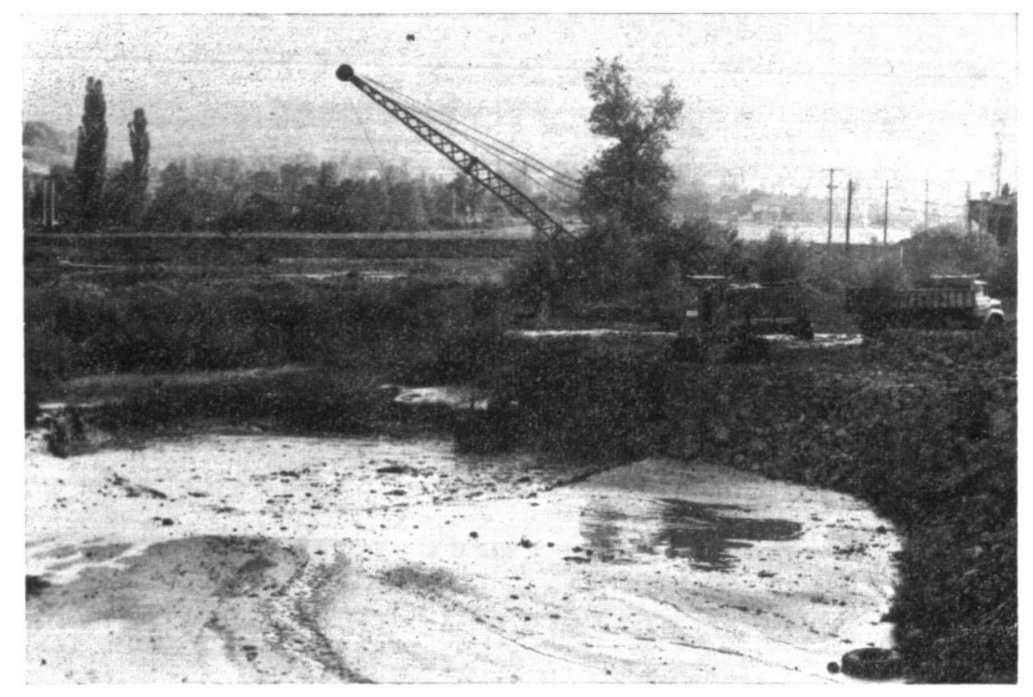

Photo 8 : CURAGE D'UN BASSIN DE DECANTATION d'eau de lavage, en bordure de Loire (Haute-Loire, été 1974).

Même avec un drag-line important, ce curage s'effectue dans de mauvaises conditions, du fait :

- de la forme défavorable du bassin, dont toutes les parties ne sont pas accessibles;

- de l'humidité excessive des boues, faute de disposer d'un deuxième bassin pour recevoir les eaux de lavage pendant une période suffisante pour le séchage du premier.

Ces excavations peuven ̀̀ d'ailleurs servir elles-mêmes à la décantation des eaux de lavage; l'eau retourne ensuite à la rivière ou à la nappe par infiltration.

De telles excavations ne sont évidemment pas disponibles dans le cas des gravières opérant uniquement dans le lit mineur. C'est donc line raison supplémentaire pour proscrire, dans toute la mesure du possible, ce type d'exploitation.

b) Problème des extractions dans le lit mineur du cours d'eau.

Etant donné l'importance des perturbations et des pollutions mécaniques qu'il entraine (remise en circulation de tonnages de MES généralement très supérieure à celle provoquée par le rejet d'eaux de lavage) et la difficulté technique à limiter ces nuisances, ce mode d'exploitation devrait être interdit en France, comme il l'est dans la piupart des pays européens. Cette règle devrait rester applicable même lorsque de gros programmes routiers nécessitent localement de très grandes quantitès de granulats : II est toujours possible de les trouver hors du lit mineur. 

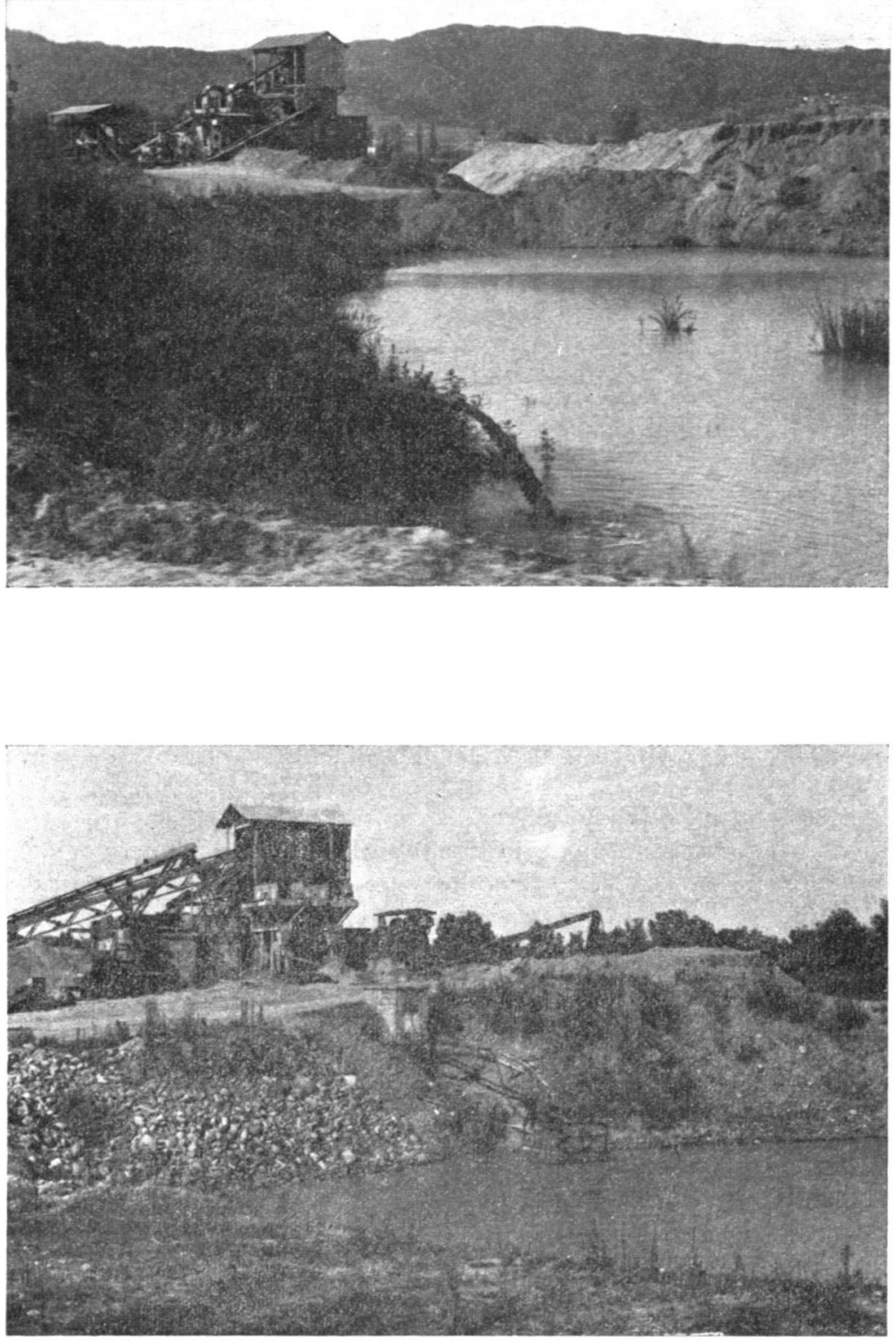

Photos 9 a et $b$ : RECYCLAGE DES EAUX DE LAVAGE, dans une exploitation sur la Loire (Ouillon, à Bas-en-Basset, Haute-Loire, été 1974) :

Photo 9 a : Rejet des eaux de lavage (1er plan) dans un trou d'ancienne extraçion où se déposent les matières les plus grossières.

Photo $9 \mathrm{~b}$ : A l'extrémité d'un deuxième bassin communicant avec le premier, l'eau est pompée pour être réutilisée pour le lavage; la crépine d'aspiration est fixée à un flotteur pour rester à distance faible et constante de la surface. Ce *lavage en circuit fermé " préserve totalement le cours d'eau de la pollution mécanique. 
Seules les opérations lièes effectivement à une lutte contre les divagations excessives du lit, ou contre les inondations, mériteraient d'être autorisées ( ${ }^{\star}$ ). Dans ce cas, il est seulement possible de limiter les dommages, non de les éviter. Pour réduire au moins la pollution mécanique en aval des chantiers, il est nécessaire alors

- de séparer la zone d'extraction du reste du cours d'eau par une digue provisoire suffisamment étanche $\left(^{* \star}\right)$;

- ou, de ne mettre les nouvelles sections du lit creusées, en communication avec le cours d'eau, que lorsque les dragages y sont terminés;

- et, enfin, de cesser toute operation susceptible d'entrainer des pollutions mécaniques dans les périodes de faible débit et/ou de forte chaleur ("**) où le cours d'eau est particulièrement vulnérable.

Ajoutons que toute exploitation dans le lit mineur d'un cours d'eau devrait de tciute façon être proscrite de certaines zones particulièrement * sensibles* telles que, notamment :

- zones de reproduction de salmonidés, notamment saumon:

- voisinage de seuils ou barrages, risquant de devenir des obstacles sérieux pour les poissons migrateurs (voir III 1).

\section{$\mathrm{VI}-$ CONCLUSIONS}

Par l'étude de quelques exemples sur la Loire supérieure et l'Allier, le présent travail a montré que, bien que n'apportant pas de substances * toxiques», au sens habituel du terme, les extractions de matériaux alluvionnaires dans les cours d'eau, ou à proximité immédiate, avaient, presque toutes, des effets incontestablement défavorables sur les communautés vivant dans ces cours d'eau $\left({ }^{* * *}\right)$. Ces nuisances sont de l'un ou des deux types suivants :

- Perturbations assez comparables à celles des aménagements hydrauliques (rectification, recalibrage), décrites ces dernières années par divers auteurs; à savoir essentiellement suppression de la diversité morphodynamique, réduisant la capacite d'accueil du nouveau lit vis-à-vis de la plupart des populations de poissons recherchées, et création d'obstacles nouveaux, également préjudiciable à certains poissons.

- Sur une distance pouvant atteindre plusieurs kilomètres à l'aval : " pollution mécanique - (turbidité accrue, colmatage du fond). Bien que s'avérant mal

$\left({ }^{*}\right)$ Les nuisances piscicoles propres à ces rectifications (et les moyens de les limiter) ont été mises en évidence, ces dernières années, dans diverses régions de france ou de Belgique (1, 11, 17, 18, 24, 29. 34). Quant à la lutte contre les inondations, il nous semble qu'elle devrait être mise en œuvre à léchelon d'un bassin fluvial plutît qu'à l'échelon dẹpartemental ou local.

$\left({ }^{* *}\right)$ Ce procédé est de plus en plus appliqué sur la majorité des exploitations sur la Dordogne, dans le département du même nom.

(**) Position adoptée dans les départements de la Dordogne et du Lot.

(***) L'étude hydrobiologique réalisée en 1975 par le SRAE Auvergne sur l'ensemble du cours de l'Allier (31) a également montré des augmentations de MES de 11 à $14 \mathrm{mg} / \mathrm{l}$ dans diverses zones d'extraction et a noté laction néfaste des différentes sablières sur le milieu naturel, dans toute la partie aval de cette rivière. 
quantifiable par la méthode des indices biotiques, cette pollution est incontestable et facile à mettre en évidence par le dénombrement des invertébrés benthiques dont la majorité des groupes faunistiques sont raréfiés. Ces groupes étant, pour la plupart, ceux qui servent de base à l'alimentation des poissons, la capacité biogénique du milieu est diminuée (alors qu'elle peut être augmentée à l'aval de pollutions organiques).

- Si on ajoute les effets observés in situ sur les œufs, ainsi que ceux observés par d'autres auteurs sur les alevins (colmatage des branchies), on explique bien les effets défavorables, sur les populations de poissons, des matières en suspension rejetees par les gravières ( $\left.{ }^{\star}\right)$.

Le mécanisme d'ensemble de ces effets sur les poissons est schématisé à la figure 9. Cette augmentation - artificielle " des matières en suspension, du fait de sa permanence et surtout de ce qu'elle occasionne des dépóts notables, a des effets incontestablement plus nocifs que celle que l'on observe normalement en crue, alors que la vitesse du courant est augmentée.

(") Les effets des matières en suspension sur la flore et la production primaire mériteraient d'étre étudiés. Leurs conséquences sur la chaine alimentaire et lauto-épuration sont probables. 


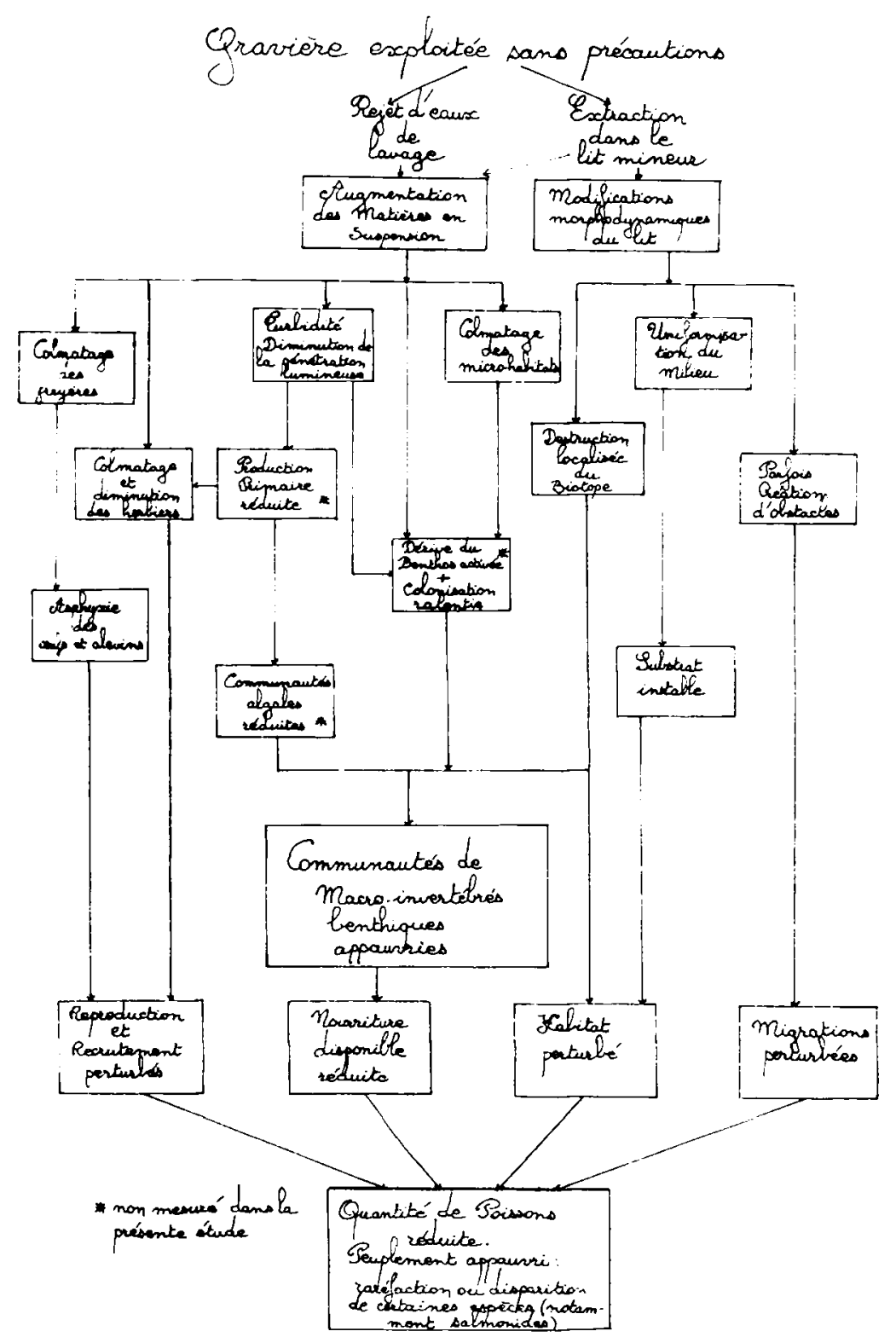

Fig. 9 : SCHEMA DU MODE D'ACTION D'UNE GRAVIERE SUR LE BIOTOPE ET SUR LES COMMUNAUTES AQUATIQUES.

Contrairement aux pollutions organiques, cette pollution mécanique se dilue vers l'aval, mais n'est pas susceptible d'auto-épuration dans le cours d'eau lui-même. Il est par contre techniquement très facile et très peu coûteux de la supprimer. Seules les extractions dans le lit même du cours posent un problème sérieux qui ne peut le plus souvent être résolu complètement que par leur suppression, au profit de chantiers plus compatibles avec la protection de l'environnement. 
II est certain que les extractions pratiquées en dehors du lit mineur du cours d'eau peuvent aussi comporter d'autres nuisances, d'ordre écologique ( ${ }^{*}$ ), hydrologique (effets sur nappes phréatiques: $3,4,23,26$ ), et esthètique (dégradation du paysage). Bien que non abordèes dans notre étude, Il nous semble que, pas plus que celles que nous avons constatées dans la rivière elle-même, ces nuisances ne sont inéluctables. Dans bien des cas, au contraire, des gravières, situées à des distances convenables des cours d'eau "sensibles*, peuvent constituer un élément positif dans l'aménagement du paysage et des loisirs de l'eau, comme on peut le constater dans quelques régions.

Dans tous les cas, une intégration harmonieuse de ces extractions dont l'utilité n'est pas contestée — dans l'ensemble des usages de i'eau, suppose :

- une réflexion préalable au choix des zones d'extraction, à l'échelon d'un département, sinon d'une région;

- une conduite des chantiers tenant compte des exigences écologiques et prévoyant le “devenir" des lieux lorsque l'exploitation sera terminée. Des suggestions pratiques ont été faites sur ce point dans la Région Piscicole de Compiègne (2).

Du fait, probablement, de l'accroissement important de la demande en matériaux de construction et de viabilité ces dernières années, ies contraintes * environnement " ont incontestablement été nég!igées, dans la plupart des exploitations de graviers sur la Loire supérieure et sur l'Allier, dans les départements de la Haute-Loire, du Puy-de-Dôme et de l'Allier.

Depuis la réalisation de cette étude, et la diffusion de ses résultats au niveau régional, une nouvelle position des administrations départementales commence à se traduire sur le terrain : choix des emplacements d'extraction et précautions prises sur les chantiers, dans un sens plus compatible avec l'environnement et les divers autres usagers du cours d'eau. L'intérêt général nécessiterait que cette évolution se confirme, notamment sur l'Allier, dernier affluent de la Loire ou se reproduit encore le Saumon.

(*) Echauffement du cours d'eau, contamination de celui-ci par des espèces indésirables, etc. étudiès notamment à la Région Piscicole de Compiègne $(5,6,25)$. 


\section{$-152-$ \\ VII - BIBLIOGRAPHIE}

1. ARRIGNON J., ROUYER G., CHANCEREL F., 1974. La sauvegarde des intérèts piscicoles au cours des travaux d'aménagement des cours deau. Compte rendu session de la SHF. La Houille Blanche no spécial 2/3 $1975: 117-125$.

2. ARRIGNON J., 1975. Aménagement piscicole des carrières humides. Bulletin d'information du Conseil Supérieur de la Pèche, 101: 33-45.

3. BABOT M., 1974. Influence des exploitations de sables et graviers sur la qualité des eaux souterraines. Techniques et Sciences municipales, 6 : 374-375.

4. BARRES M., 1974. La pollution des eaux souterraines par les décharges. Techniques et Sciences municipales, $6: 365-371$.

5. BURLAT M.F., 1975. Les Ballastières dans la vallée de la Risle. Bulletin d'information du CSP, $101:$ 21-32.

6. BRGM, 1975. Etude de l'impact thermique de ballastieres situées près de cours d'eau de 1 re catégorie piscicole. Compte rendu réunion service des Mines, Rouen : 1-4.

7. BRGM, CTE, 1975. Val d'Allier. Synthèse des ressources en eau et en granulats dans les départements du Puy-de-Dôme et de l'Allier.

8. ClaVel P., MARTY A., CUINAT R., 1975. Qualité de l'eau de la Loire en Haute-Loire. Premiers résultats concernant les effets d'extractions de matériaux alluvionnaires sur le milieu aquatique $\left({ }^{*}\right)$. Polyc. : $15 p$.

9. CLAVEL P., 1975. Contribution à l'étude écologique du cours supérieur de la Loire et de quelques affluents dans le departement de la Haute-Loire. Les effets des extractions de matériaux alluvionnaires dans le lit de la Loire, sur la macrofaune d'invertébrés benthiques. DEA de Biologie Animale $\left({ }^{\star}\right)$. Polyc. : $75 \mathrm{p}$.

10. ClaVel P., haMON Y., ROMANEIX C., 1976. Effets des extractions de matériaux alluvionnaires sur l'environnement aquatique dans les cours supérieurs de la Loire et de l'Allier. Thèse de Doctorat 3 e cycle et d'Ingéniorat $\left(^{*}\right)$. Polyc. : $197 \mathrm{p}$.

11. CTGREF, 1976. Effets biologiques et écologiques des extractions de matériaux dans le lit des cours d'eau (Pollution a mécanique"). Rappoit préliminaire. Observations réalisées sur le cours inférieur du Doubs, no 12 , Antony : $1-26$.

12. CUINAT R., 1971. Ecologie et Repeuplement des cours d'eau à truites. Bull. Fr. Piscic., 240-242-243: $87 \mathrm{p}$.

13. CUINAT R., 1974. Les exigences des poissons dans nos eaux courantes. La Technique de l'eau et de l'assainissement, $350: 1-31$.

${ }^{*}$ Cons. Sup. de la Pêche, Rég. Pisc. Auvergne-Limousin. 
14. CUINAT R., 1976. Principaux facteurs menaçant la survie du saumon LoireAllier. CSP, Rég. Pisc. *Auv.-Lim.", Polyc. : 11 p.

15. FAO, 1970. Critères de qualité de l'eau; III : poissons, autre vie aquatique et faune sauvage. Techn. paper $94: 118 \mathrm{p}$.

16. GAMMON J.R., 1970. The effect of inorganic sediment on stream biota. U.S. Environmental Protection Agency, Water Pollution control Research Series. 18050 DWC 12/70: $141 \mathrm{p}$.

17. GEBHARDS S., 1973. Effect of channelization on fish, Trout Magazine, 14, $1: 22-27$.

18. HUET M. et TIMMERMANS J.A., 1976. Influence sur les populations de poissons des aménagements hydrauliques de petits cours d'eau assez rapides. Trav. Stat. Eaux et Forêts, Sèr. D, $46: 27$ p.

19. HYNES H.B.N., 1960. Biology of Polluted Waters. Liverpool University Press.

20. INSEE, 1971. Statistiques BIC.

1973. Statistiques Industrielles.

1973. Statistiques de l'Industrie Minérale.

1973. Les Structures Industrielles Françaises.

"Matériaux de construction et industries connexes *

Ministère de l'Industrie et de la Recherche.

1974. Tableaux de l'Economie Régionale - Auvergne.

21. KHALAF G., 1975. Utilisation de substrats artificiels, en eau courante, pour l'étude de la répartition et de la dynamique de colonisation des macroinvertébrés benthiques. Thèse de 3 e cycle, UER des Sciences de la Nature, Lyon I : 91 p. + Tab. et Fig.

22. LEGER L., 1910. Principe de la méthode rationnelle du peuplement des cours d'eau à salmonidés. Trav. Lab. Piscic. Univ. Grenoble, 1 : 531-602.

23. PEAUDECERF P., 1974. Effets des gravières sur le comportement Hydrodynamique des nappes d'eau souterraines. Compte rendu session SHF, La Houille Blanche, 2-3: 133-140.

24. PHILIPPART J.C., 1976. Les effets biologiques du curage mécanique et de la rectification des rivières. La Gazette Officielle de la Pêche, 553.

25. PORCHER J.P., 1975. Déséquilibres écologiques liés à l'implantation de ballastières sur les cours d'eau de 1 re catégorie piscicole, Communication à l'Ass. Fse. Limn., CSP tre Région Piscicole.

26. PRUDHOMME P., 1975. Aménagement du Var inférieur et protection des nappes souterraines : un exemple d'extraction contrôlée de graviers. Compte rendu Session SHF ; La Houille Blanche 2-3 : 145-153.

27. ROMANEIX C., HAMON Y., GARBAY V., DESFARGES L., 1975. Influence d'une pollution mécanique sur la faune benthique. Mémoire $3 e$ année ESITPA, Polyc. : 55 p. $\left({ }^{\star}\right)$

28. ROSENBERG D.M., WIENS A.P., 1975. Experimental sediment addition studies on the Harris River, NWT, Canada: The effect on macroinvertebrate drift. Verh. Internat. Verein. Limnol., $19:$ 1568-1574.

(*) Cons. Sup. de la Pêche, Rég. Pisc. Auvergne-Limousin. 
29. ROUYER G., 1975. Entretien des rivières et aménagements hydrauliques. Le courrier de la Nature, $39: 218-230$

30. SHANNON C.E., WEAVER W., 1949. The mathematical theory of communication. Urbanna, Univ. III Press. 1963 ed., 125 p.

31. SRAE Auvergne, 1975. Etude de l'Allier. Polyc. : $17 \mathrm{p}$. + Tab. et Annexes.

32. SHERK J.A., 1971. The effects of suspended and deposited sediments on estuarine organisme. Chesapeake Biological Laboratory, Solomons, Maryland.

33. TUFFERY G., VERNEAUX J., 1968. Mèthode de détermination de la qualité biologique des eaux courantes, CERAFER Paris : $21 \mathrm{p}$.

34. VERNEAUX 1., VERGON J.P., LAR:NIER M., 1974. Aspects écologiques des travaux d'aménagement des cours d'eau. Orientations et principes généraux. Compte rendu Session SHF, La Houille Blanche, 2-2 : 127-132.

35. VERNEAUX J., LEYNAUD G., 1974. Introduction à la définition * d'objectifs *, puis de "critères", de la qualité des eaux courantes. CTGREF, Division Qualité des Eaux, Pêche et Pisciculture, Paris, 28 p.

36. VIBERT R., LAGLER K.F., 1961. Pêches Continentales. Biologie et aménagement, 1 vol. : 720 p. Paris, Dunod.

37. VIBERT R., 1975. Repeuplements des eaux à truites. La Pisciculture Française, 42 : 25-48.

38. WOODIWISS F.S., 1964. The biological system of Stream classification used by the Trent River Board. Chemistry and Industry : 443-447. 